\title{
Induction of the ALT pathway requires loss of ATRX- DAXX in concert with genotoxic lesions at telomeres
}

David Clynes ( $\boldsymbol{D}$ david.clynes@oncology.ox.ac.uk)

University of Oxford

\section{Tomas Goncalves}

University of Oxford https://orcid.org/0000-0002-3342-0461

Thomas Kent

University of Oxford

\section{Sam Shepherd}

University of Oxford

Siobhan Cunniffe

University of Oxford

\section{Sangwoo Kim}

University of Oxford https://orcid.org/0000-0002-2951-3331

\section{Tim Humphrey}

University of Oxford

Richard Glbbons

University of Oxford

\section{Article}

Keywords: ATRX, Glioma, Alternative lengthening of telomeres, Cancer, SETD2, DAXX, R-loops, Break Induced Replication

Posted Date: April 28th, 2021

DOI: https://doi.org/10.21203/rs.3.rs-362334/v1

License: (c) (i) This work is licensed under a Creative Commons Attribution 4.0 International License. Read Full License 
1 Induction of the ALT pathway requires loss of ATRX-DAXX in concert with genotoxic lesions at

2 telomeres

3

4 Tomas Goncalves ${ }^{1,3 \#}$, Thomas Kent ${ }^{1,3 \#}$, Sam Shepherd ${ }^{1,2}$, Siobhan Cunniffe ${ }^{1,2}$, Sangwoo Kim ${ }^{1,2}$,

5 Timothy C. Humphrey², Richard J. Gibbons ${ }^{3}$, David Clynes ${ }^{1,2^{*}}$

6

7

${ }^{1}$ MRC Weatherall Institute of Molecular Medicine, University of Oxford, John Radcliffe 8 Hospital, Oxford OX3 9DS, UK.

9

$10{ }^{2}$ MRC Oxford Institute for Radiation Oncology, Department of Oncology, University of Oxford, 11 Oxford OX3 7DQ, UK

$12{ }^{3}$ MRC Molecular Haematology Unit, MRC Weatherall Institute of Molecular Medicine,

13 University of Oxford, John Radcliffe Hospital, Oxford OX3 9DS, UK

14 \#These authors contributed equally to this work

15

16 *Correspondence: david.clynes@oncology.ox.ac.uk Tel: 00441865222440

17

18 
21 A key requisite for indefinite growth of cancer cells is the ability to continuously elongate

22 telomeres to circumvent the onset of senescence or apoptosis. In approximately $10-15 \%$ of

23 cancers this is achieved through the Alternative Lengthening of Telomeres (ALT) pathway, a

24 Break Induced Replication (BIR) mediated mechanism of telomere copying. ATRX has

25 emerged as the key tumour suppressor in ALT cancers but its loss is insufficient to drive

26 induction of the pathway. Here, we report that depletion of ATRX and/or DAXX in the

27 presence of various genotoxic agents is sufficient to induce ALT. Moreover, co-deletion of

28 ATRX and SETD2, commonly mutated in high grade gliomas (HGGs), elicits induction of ALT.

29 Mechanistically, SETD2 restricts the accumulation of telomeric R-loops, which, in the absence

30 of ATRX, leads to fork collapse and the loss of telomere sister chromatid cohesion.

31 Cumulatively this provides a substrate for out of register BIR and telomere lengthening.

33 KEYWORDS

34 ATRX, Glioma, Alternative lengthening of telomeres, Cancer, SETD2, DAXX, R-loops, Break 35 Induced Replication 
37 Due to the inherent inability of DNA polymerases to fully replicate the distal ends of linear chromosomes, chromosomal DNA is progressively shortened with each round of cell division.

39 To circumvent potentially detrimental effects to genome stability and loss of genetic information, humans, like many other species, have developed specialised nucleoprotein structures, called telomeres, that are comprised of many kilobases $(\mathrm{kb})$ of a tandem repeat sequence, TTAGGG, culminating in a 3' protrusion of single stranded G-rich DNA of $50-400$ nucleotides. Telomeric sequence is bound by a specialised protein complex, denoted Shelterin, which comprises the proteins TRF1, TRF2, POT1, TIN1, TPP1 and RAP1 ${ }^{1}$. Telomeres range from 3 to $12 \mathrm{~kb}$ in humans and progressively shorten by about 200 base pairs per cell division due to the end-replication problem. Once telomeres reach a critical length, termed the 'Hayflick limit', they elicit DNA damage checkpoint activation, leading to cellular senescence or telomere-induced apoptosis. A key hallmark of cancer cells is their ability to circumvent telomere shortening via a telomere length maintenance mechanism (TMM). In the majority of cancers, this is achieved through the upregulation of telomerase, a specialised ribonucleoprotein that acts to progressively add telomeric repeats to the end of chromosomes. More recently it has emerged that approximately $15 \%$ of cancers maintain telomere length through a telomerase independent TMM, known as the Alternative Lengthening of Telomeres (ALT) pathway. The ALT pathway is particularly prevalent in cancers of mesenchymal origin, including several cancers of the central nervous system (CNS), with rates as high as $63 \%{ }^{2}$. ALT in human cancer cells is generally considered to be a form of aberrant telomere recombination and conservative DNA synthesis known as Break Induced Replication (BIR), occurring during both the $\mathrm{G} 2$ and Mitosis phases of the cell cycle. A threeprotein axis has been implicated in facilitating ALT-mediated telomere synthesis; POLD3, PCNA and RAD52 ${ }^{3-5}$. A second Rad52 independent pathway has also recently been reported, 
61 suggesting that ALT is in fact a bifurcated pathway, with similarities to telomerase independent telomere maintenance pathways originally described in budding yeast ${ }^{6,7}$.

63

64 Further work in human cells has suggested that replication stress arising at telomeres can potentiate the ALT pathway ${ }^{4}$. Indeed, owing to their repetitive nature, telomeres are thought to be inherently difficult sequences to replicate and telomeres have been shown to phenotypically resemble common fragile sites due to the overt fragility they exhibit under conditions of replication stress ${ }^{8}$. This is likely in large part due to the propensity of the G-rich repetitive telomeric sequence to adopt non-canonical DNA secondary structures, including the G-quadruplex (G4) conformation and R-loops; three-stranded nucleic acid structures consisting of an RNA:DNA hybrid and a displaced piece of single stranded DNA ${ }^{9}$. In line with this notion, abrogation of FANCM activity, which has known roles in R-loop resolution and fork stabilisation ${ }^{10-12}$, or depletion of RNase $\mathrm{H}$, which can degrade DNA:RNA hybrids, have both recently been shown to potentiate markers of the ALT pathway $10,13,14$

A near unifying feature of ALT positive cancer cells is loss of the alpha thalassemia/mental retardation syndrome X-linked chromatin remodeller (ATRX) and/or its interaction partner DAXX (death domain-associated protein) ${ }^{2,15,16}$. Indeed, previous work by our lab and others has demonstrated that ectopic expression of ATRX leads to a DAXX-dependent suppression of the ALT pathway ${ }^{17,18}$. Of note, depletion or knockout (KO) of ATRX in telomerase positive or primary cell lines is generally insufficient to induce markers of the ALT pathway ${ }^{15,17,19,20}$, with the notable exception of a minority of glioma cell lines ${ }^{21}$. A likely explanation for these observations is that other genetic or epigenetic events are required for the induction of ALT 
84 in human cancer in concert with ATRX loss. To date, what these events are remains

85

86

87 contentious.

ATRX is a chromatin remodelling factor of the Snf2 family (reviewed in ${ }^{22}$ which, together with the histone chaperone DAXX, facilitates the incorporation of the histone variant H3.3 into defined genomic sites, including telomeric chromatin, in a replication independent chromatin assembly pathway ${ }^{23-25}$. In the last decade, a number of studies have implicated ATRX in a plethora of roles related to the maintenance of genome stability. One major role of ATRX appears to be the regulation of non-canonical DNA secondary structures, with ATRX null cells displaying increases in both $\mathrm{G} 4$ and R-loop structures ${ }^{26,27}$. ATRX also has multiple reported roles in DNA replication; including the prevention of replication fork stalling, potentiation of fork restart and the prevention of excessive nucleolytic degradation of stalled forks ${ }^{20,28-30}$. Additionally, evidence exists for a role of ATRX in facilitating DNA double strand break (DSB) repair, with roles reported in both the major DSB repair pathways, homologous recombination (HR) ${ }^{31,32}$ and non-homologous end joining (NHEJ) ${ }^{33}$. Which of these role(s) are required for ATRX to prevent either the induction or maintenance of the ALT pathway is to date unclear.

Here, we report on an overarching role for ATRX in the suppression of the ALT pathway. Firstly, we demonstrate that ATRX/DAXX is required to prevent ALT in the presence of a variety of genotoxic agents that are known to result in replication fork stalling and reversal. Induction of the ALT phenotype is associated with increases in telomeric DNA damage and single stranded DNA, in addition to a loss of sister chromatid telomere cohesion. Based on this we propose that the induction of ALT in cancer cells requires two independent events; 

one involving a mutation (or mutations) in a factor (or factors) that leads to the generation of

109 replicative stress at telomeres, which, if in concert with ATRX loss, leads to both fork collapse

110 and the loss of telomere sister chromatid cohesion. This cumulatively results in out of register

111 BIR and telomere lengthening. We demonstrate one such factor to be the histone

112 methyltransferase SETD2, which is frequently mutated concomitantly with ATRX in high grade

113 gliomas (HGGs), by showing that loss of SETD2 elicits induction of the ALT pathway,

114 specifically in the absence of ATRX, via the generation of telomeric R-loops.

115

116 
121 Previous work has reported that depletion or KO of ATRX in telomerase positive or primary 122 cell lines is insufficient to induce markers of the ALT pathway $15,17,19,20$, with the notable exception of a minority of glioma cell lines ${ }^{21}$. One implication of these findings is that the loss

124 of ATRX, while generally a requisite for ALT pathway induction and/or maintenance, must occur in concert with other genomic events within cancer cells that cumulatively lead to telomere lengthening via ALT. We therefore sought to explore this possibility further.

The HeLa long telomere cell line (henceforth referred to as HeLa LT) is a subclone of HeLa with

129 long telomeres ( $20 \mathrm{~kb}$ ) which has previously shown to be amenable to ALT induction through

130 depletion of ASF1 ${ }^{34}$. Consistent with previous reports, two independent ATRX CRISPR-Cas9

131 KO HeLa LT clones (Figure 1A) conferred sensitivity to a variety of genotoxic agents known to 132 induce replicative stress, including the G4 stabilising ligand pyridostatin (PDS), aphidicolin $133(\mathrm{APH})$ and hydroxyurea (HU) (Figures S1A-C). Despite these conferred synthetic lethal 134 interactions, neither of the KO clones elicited an increase in cardinal ALT markers, including 135 C-circles (Figures 1B and 1C), ALT-associated PML nuclear bodies (APBs) (Figures 1D and 1E) or telomere length heterogeneity (Figure 1F). 
141 in the absence of ATRX

142

143 A key feature of telomeres is their propensity to adopt non-canonical DNA secondary

144 structures, including the G4 conformation, which has been implicated in both transcriptional 145 dysregulation and DNA damage ${ }^{35}$. ATRX binds widely at sites in the genome predicted to form

146 G4 structures, including telomeres ${ }^{25,36}$ and ATRX loss confers sensitivity to various G4

147 stabilising ligands, including PDS (Figure S1A), suggesting that ATRX protects G4 sites from

148 genome instability ${ }^{26,30}$. In accordance with this, we observe that the addition of PDS led to

149 an increase in ATRX recruitment to telomeres (Figures S2A and S2B), suggestive of a putative

150 role for ATRX in protecting telomeric DNA upon the stabilisation of G4s. To explore this

151 possibility further, we treated our two HeLa LT KO clones with PDS and assessed for markers

152 of activation of the ALT pathway. Strikingly, treatment with PDS elicited an increase in many

153 of these cardinal markers, including C-circles (Figures 2A and 2B) and the formation of APBs

154 (Figures 2C and 2D) to levels comparable to the archetypical ALT cell line U-2 OS, whereas no

155 notable increase was observed in the ATRX wildtype cells. Addition of PDS also elicited a

156 decrease in total telomere number and an increase in telomere intensity, consistent with

157 increased telomere clustering and telomere synthesis (Figures S3A and S3B).

159 ALT has been suggested to be coincident with telomeric replicative stress, with an 160 accumulation of RPA2 at telomeres upon ALT induction ${ }^{34}$ and an increase in single stranded 161 telomeric DNA (ssTel), as detected by non-denaturing telomeric FISH ${ }^{37}$. Consistent with these 162 findings, an ALT specific co-localisation between RPA and ssTel is clearly detectable across a 163 panel of cell lines (Figures S4A and S4B). This signal was refractory to RNase A and RNase $\mathrm{H}$ 
treatment, confirming that the ssTel signal was detecting telomeric DNA and not TERRA RNA

165 (Figure S4C). Inducible expression of ATRX in ALT positive U-2 OS cells, using our previously 166 characterised U-2 OS ${ }^{A T R X}$ cell line ${ }^{18}$ led to a complete abrogation of RPA ssTel foci, once again

167 confirming RPA/ssTel as a bona-fide ALT marker and suggesting that the signal is a 168 consequence of ATRX loss in ALT cancer cells (Figure S4B). We next asked whether treatment 169 of our Hela LT ATRX KO clones with PDS also induced formation of this novel ALT marker. As 170 expected, addition of PDS significantly increased detectable RPA ssTel foci in both 171 independent ATRX KO clones, with no detectable increase in wildtype ATRX HeLa LT cells

172 (Figures $2 \mathrm{E}$ and $2 \mathrm{~F}$ ). ALT positive cell lines characteristically exhibit long and heterogeneous telomere lengths ${ }^{38}$. Consistent with a bona fide induction of the ALT pathway, treatment with PDS led to a characteristic lengthening and spread of telomere lengths in the ATRX KO clones but not in the ATRX wildtype cells (Figure 2G). We also used monochrome multiplex qPCR (mm-qPCR) analysis to show that the $\mathrm{T} / \mathrm{S}$ ratio - the telomere repeat number normalised to the single copy gene $H B B$ (encoding beta-globin) - significantly increased after PDS treatment in both ATRX KO clones but not in the wildtype cells (Figure S5A), again highly suggestive of a bona fide induction of the ALT pathway and associated telomere synthesis in these cells.

Induction of ALT in the absence of ATRX upon PDS treatment is dependent on the Mus81 endonuclease

It has been hypothesised that ALT emanates from the formation of a DSB within the telomere sequence, likely attributable to the stalling, reversal, and subsequent collapse of replication forks. Consistent with an induction of an ALT like process and fork collapse events, loss of

187 ATRX was associated with a significant increase in telomere-associated ATM, 
autophosphorylated at serine 1981 (pATM S1981), a marker of ATM activation at DNA DSBs

${ }^{39}$ (Figures $3 \mathrm{~A}$ and $3 \mathrm{~B}$ ). The cleavage of stalled forks to generate a DSB is dependent on the

190 activity of the structure-selective endonuclease MUS81-EME2 ${ }^{40}$ and, in agreement with a

191 central role for replication fork collapse in the initiation of ALT, MUS81 activity is a requisite

192 for telomere maintenance in ALT cells ${ }^{40,41}$. We next sought to explore whether the

193 destabilisation and collapse of replication forks was a feature of ALT induction upon the

194 addition of PDS to our ATRX KO cells. Consistent with this requirement, knockdown of Mus81

195 completely abrogated the increase in C-circles observed in the ATRX KO cells upon PDS

196 treatment (Figures 3C and 3D). Likewise, the depletion of Mus81 was also associated with an

197 attenuation of RPA ssTel foci in the ATRX KO cells (Figures 3E and 3F). Taken together, we

198 conclude that the generation of ALT upon ATRX loss and PDS treatment is likely attributable

199 to an increased destabilisation and Mus81-dependent processing of replication forks in the absence of ATRX, which in turn generates the DSB substrate for subsequent BIR-mediated lengthening of telomeres.

Genotoxic agents that cause replicative stress induce markers of ALT in ATRX deficient cells

Recent work has shown that replication fork uncoupling and reversal is a global response to a plethora of genotoxic agents that can cause adducts or lesions on DNA ${ }^{42}$. Given the reported roles for ATRX in promoting replication fork processivity and stability ${ }^{20,28,29}$, we considered the possibility that the addition of a panel of genotoxic agents at sublethal doses, which on their own are reported to induce fork remodelling but not collapse, could also specifically elicit ALT in the absence of ATRX. Perturbations in fork processivity and reversal have been

211 extensively reported as a frequent event upon addition of the Topoisomerase I inhibitor 
camptothecin (CPT) ${ }^{43}$. In agreement with the conjecture that ATRX is required to protect

213 telomeres under conditions of replicative stress, addition of a low dose of CPT induced the

214 formation of C-circles to levels almost equivalent to those found in the ALT positive U-2 OS

215 cell line (Figure 4 A). Likewise, addition of CPT led to an increase in APBs (Figures 4B and 4C)

216 and RPA ssTel foci (Figures 4D and 4E) as well as a significant increase in telomere length

217 heterogeneity (Figure S5B), specifically in the ATRX KO clones, consistent with a bona fide

218 induction of the ALT pathway. Interfacial inhibition of Topoisomerase II, through addition of

219 etoposide (ETO), elicited a similar response, with preferential increases in C-circles (Figure

$2204 \mathrm{~A}$ ), APBs (Figures 4B and 4C) and RPA ssTel foci (Figures 4D and 4E) in ATRX deficient cells, albeit to a slightly lesser extent than that observed with CPT. Treatment with low doses of the DNA polymerase inhibitor APH, which has previously been shown to generate replicative stress at common fragile sites ${ }^{8}$, elicited only a minimal increase C-circles which failed to reach significance (Figure 4A) and no detectable increase in APBs (Figures $4 B$ and $4 C$ ) in the ATRX null cells, but surprisingly did lead to a notable increase in RPA ssTel foci (Figures 4D and 4E). Taken together, this data suggests that it is the formation of a lesion or structure on telomeric DNA, and not replication stress per se, that facilitates induction of the ALT pathway in the absence of ATRX.

To assess whether induction of ALT was specific to the HeLa LT cell line upon loss of ATRX, we sought to deplete ATRX via shRNA in a non-LT HeLa cell line with standard length telomeres (HeLa ST) (Figure S6A). Consistent with our previous observations in the HeLa LT cell line, addition of camptothecin (CPT), specifically in ATRX depleted cells, led to a robust augmentation of C-circles (Figures S6B and S6C), albeit to a lesser extent than that observed in the HeLa LT ATRX KO clones. Likewise, we found that addition of PDS led to induction of 
another of the ALT hallmarks, the RPA ssTel foci, again only in the ATRX depleted cells (Figures

237 S6D and S6E). Taken together, we conclude that the observed induction of ALT is not specific

238 to the HeLa LT cell line, however, the presence of long telomeres does appear to exacerbate 239 the response.

ATRX and DAXX function epistatically in the suppression of $A L T$

A minority of ALT cancers are characterised by a loss of the histone chaperone protein DAXX

which, together with ATRX, forms a complex that can facilitate incorporation of the histone variant H3.3 into ribosomal ${ }^{44}$, pericentromeric ${ }^{23}$ and telomeric ${ }^{24,25}$ chromatin. We have previously reported that the suppression of the ALT pathway through ectopic expression of ATRX is lost upon depletion of DAXX ${ }^{18}$, suggesting that ATRX and DAXX likely work in concert in the suppression of ALT. To further explore the relationship between ATRX and DAXX in the suppression of the ALT pathway, CRISPR-Cas9 mediated DAXX KO clones in the HeLa LT cell line, both in the context of WT ATRX and ATRX KO, were generated (Figure 5A). Consistent with the notion that ATRX/DAXX function as a complex in the suppression of the ALT pathway, the loss of DAXX facilitated the accumulation of C-circles (Figures 5B and 5C), RPA ssTel foci (Figures 5D and 5E) and APBs (Figures 5F and 5G) upon treatment with both PDS and CPT. Importantly, the co-depletion of both ATRX and DAXX failed to confer any cumulative increase in these cardinal ALT markers (Figures $5 B-G$ ), strongly suggesting that both ATRX and DAXX are functioning within the same pathway in the suppression of telomeric replicative stress and the ALT pathway. 
262 Data presented here suggests that the induction of ALT in cancer cells in fact requires two independent events, involving mutations or factors that generate a telomeric structure or lesion, which, if in concert with ATRX loss, leads to fork collapse. This thereby provides the substrate and means for BIR and subsequent telomere lengthening.

Recurrent mutations in genes that disrupt the post-translational modification of histone $\mathrm{H} 3$ at $\mathrm{H} 3 \mathrm{~K} 36$, including mutations in H3.3 G34R/V or the histone methyl transferase SETD2, are also frequently found in paediatric HGGs and are highly co-incident with mutations in ATRX ${ }^{45}$. Of note, mutations in SETD2 have recently been linked to the generation of replicative stress ${ }^{46}$. To explore the possibility that loss of ATRX, in the context of SETD2 loss, is sufficient to drive the ALT pathway, we generated SETD2 KO clones both in the context of wildtype

273 ATRX and ATRX loss. Loss of SETD2 activity in the resulting clones was confirmed by a global loss of histone H3 K36me3 (Figure 6A). Loss of SETD2 alone was insufficient to elicit markers of ALT, however strikingly, the combination of both ATRX and SETD2 loss induced an accumulation of C-circles (Figures 6B and 6C), APBs (Figure 6D) and RPA ssTel foci (Figure 6E) akin to those observed in U-2 OS cells. The loss of SETD2 was associated with increases in DNA damage markers, including pATM, which were further exacerbated upon the combined KO of both SETD2 and ATRX (Figure 6F). Strikingly, this accumulation of C-circles was dramatically reduced upon treatment with the transcription inhibitor triptolide in a dose dependent manner (Figures 6 G and S7A). We noted that treatment with triptolide elicited a minor effect on cell cycle distribution, with a slight increase in the percentage of cells in G1, however, in 
doses of triptolide elicited an identical change to the cell cycle distribution (Figures S7B-F). Taken together, it is therefore unlikely that the reduction in C-circles is an indirect consequence of changes to the cell cycle. Given this dependence on transcription and previous findings that the ALT pathway is potentiated through the formation of telomeric Rloops $10,12,13,47$, we asked whether the depletion of SETD2 was associated with an increase in telomeric R-loops. Strikingly, immunoprecipitation using an antibody specific to RNA-DNA hybrids (S9.6) showed a marked accumulation of telomeric R-loops upon loss of SETD2 (Figure $6 \mathrm{H})$. When cells were pre-treated with RNase $\mathrm{H}$ prior to immunoprecipitation, the signal was decreased, suggesting that the signal was indeed specific to the DNA-RNA hybrids (Figure 6H). We next sought to determine whether the accumulation of telomeric R-loops observed upon SETD2 loss was responsible for facilitating the accumulation of ALT markers in the combined ATRX SETD2 KO cells. To this end, we overexpressed the RNA-DNA endonuclease RNase H1 in HeLa LT SETD2 KO cells (Figure 6I) which led to a marked decrease in telomeric R-loops (Figure

297 6J). Moreover, overexpression of RNase H1 in the ATRX SETD2 KO cells resulted in a reduction of C-circles that was not observed upon overexpression of a catalytically dead mutant of

299 RNase H1 (D210N) (Figure 6K). Taken together, this data supports a model whereby loss of 300 SETD2 leads to the accumulation of telomeric R-loops which triggers the ALT pathway, 301 specifically in the absence of ATRX.

ATRX is required for telomere sister chromatid cohesion pathway ${ }^{3,48}$ and the use of a sister telomere as an in-register template will not result in a net elongation of telomeres. Prior reports have suggested that loss of ATRX leads to a diminution 
308 of telomere cohesion ${ }^{19,49}$, suggesting that ATRX may have an additional major role in the

309 suppression of the ALT pathway, by ensuring the correct register of telomeric BIR. To this end,

310 we sought to determine whether loss of ATRX in the HeLa LT cell line elicited a loss of

311 telomeric cohesion using a FISH probe specific to the subtelomeric q arm of chromosome 13

312 (and an internal control probe mapping to 13q14). A loss of telomere cohesion was then

313 scored in interphase cells as the appearance of a doublet as opposed to a single focus, with

314 doublets indicative of a separation of sister telomeres. Strikingly, loss of ATRX resulted in a

315 significant increase in the proportion of doublets, consistent with a premature loss of

316 telomere sister chromatid cohesion in the absence of ATRX (Figures 7A and 7B). No significant

317 changes were observed when using a FISH probe targeting an internal region of chromosome

31813 , suggesting that defective sister chromatid cohesion in the absence of ATRX was confined

319 to telomeres (Figures 7A and 7B).

320

321 DISCUSSION

322

323 Loss of ATRX or its functional interaction partner DAXX are hallmarks of the vast majority of

324 ALT cancer cells but, despite ectopic expression of ATRX being sufficient to suppress the 325 pathway in ALT cells ${ }^{17,18}$, the loss of ATRX is generally insufficient to induce markers of the 326 ALT pathway ${ }^{15,17,19,20}$. Of note, recent work has shown that CRISPR-Cas9 mediated depletion 327 of ATRX induces canonical markers of ALT in two specific glioma cell lines (U-251 and UW479) 328 and a prostate cancer cell line (LAPC-4) ${ }^{21,50}$. Given that this induction was specific to these 329 cell lines, it is likely that additional genetic or epigenetic events occur alongside ATRX loss to 330 facilitate induction of ALT in human cancer. Consistent with this prediction, we report here 331 that the loss of ATRX in HeLa cells failed to induce the canonical markers of ALT. It has been 
suggested that the presence of telomeric replicative stress drives the ALT pathway, with the

333 stalling and collapse of replication forks at telomeres leading to the generation of a one ended

334 DSB which is subsequently repaired by BIR in G2 or mitosis (MiDAS) ${ }^{4,7}$. Loss of ATRX has

335 previously been linked to the generation of replicative stress $20,28-30,51$, however, given that

336 loss of ATRX is generally insufficient to induce ALT, we reasoned that the parallel induction of

337 telomeric replicative stress, which would normally be tolerated through the activity of

338 ATRX/DAXX, could trigger the ALT pathway only when in concert with ATRX loss. In

339 concordance with this notion, we show here that the treatment of ATRX deplete cells with

340 genotoxic agents known to induce structures/lesions on DNA triggers multiple canonical

341 markers of an active ALT pathway, specifically in the absence of ATRX and/or DAXX. Genotoxic

342 agents that were found to induce ALT included the G4 stabilising ligand PDS, and the topoisomerase inhibitors ETO and CPT, both of which are frequently used in cancer therapy.

344 Treatment with relatively mild doses of these drugs has been linked to fork slowing and

345 reversal in the absence of chromosomal breakage ${ }^{42}$, implying that under these conditions

346 fork collapse is facilitated only upon ATRX and/or DAXX loss. In line with this, telomeric S1981

347 phosphorylated ATM was dramatically enriched at telomeres upon PDS treatment in ATRX

348 null cells and the generation of ALT markers was dependent on the MUS81 endonuclease,

349 suggesting that forks are cleaved and collapsed in the absence of ATRX upon PDS treatment.

350 Importantly, we report that low dose APH treatment did not elicit the same response,

351 implying that it is not replicative stress per se that elicits ALT in the absence of ATRX and/or

352 DAXX, but more likely the presence of a 'lesion' or 'secondary structure' on telomeric DNA.

353 Indeed, G4 structures have been proposed to form a barrier to the replisome, although in the

354 case of telomeres the G4 structure would form on the lagging strand, which presumably

355 constitutes less of a hindrance to the fork. In line with this, it has been reported that a block 
in lagging strand replication does not result in fork arrest in vitro, but rather is bypassed via

357 re-priming by Pol $\alpha /$ Primase allowing the fork to progress ${ }^{52}$. One potential explanation for our findings is that the stabilisation of G4 structures via PDS treatment has also been shown to result in a further potential barrier to replication fork processivity in the form of R-loops,

360 with both PDS-mediated DNA damage and micronuclei formation abrogated upon the overexpression of RNase $\mathrm{H} 1{ }^{53}$. Consistent with an important role for R-loops in facilitating

362 ALT, overexpression of RNase $\mathrm{H} 1$ in ALT cells has been shown to abrogate ALT markers, whereas the loss of RNase H1 or FANCM (a factor also linked to the prevention of R-loop formation), have the opposite effect ${ }^{10-13}$.

Recent work has also suggested that PDS induced cytotoxicity is dependent on TOP2 ${ }^{54}$, 367 inferring that the formation of G4 and/or R-loop structures leads to trapping of topoisomerases which, in turn, may block replication fork progression ${ }^{55}$. We show here that, analogous to PDS, treatment with the TOP2 poison ETO elicited markers of ALT specifically in ATRX deplete cells. We note the largest induction of ALT markers manifested following treatment with the TOP1 inhibitor CPT. Interestingly, poisoning of TOP1 by camptothecins also effectively leads to the formation of R-loops ${ }^{56,57}$. We show here that the induction of Ccircles in ATRX null cells upon CPT treatment is most pronounced in the HeLa LT cell line, which harbours very long telomeres of $\sim 20 \mathrm{~kb}$ in length. This is consistent with previous findings that the induction of ALT was most robust in this cell line upon co-depletion of ASF1a and ASF1 $b^{58}$. The most likely explanation for this is that longer telomeres inherently provide more opportunity for aberrant structures and/or genotoxic lesions to form. Indeed, each telomeric repeat has been shown to represent a potential TOP1 cleavage site ${ }^{59}$. 
Strikingly, we further report here that the combined depletion of ATRX and the histone H3

K36me3 methyltransferase SETD2 triggers a dramatic induction of ALT pathway markers, akin

382 to that observed upon CPT treatment. Mutations in SETD2 have been identified in 15\% of paediatric HGGs and are highly coincident with ATRX loss ${ }^{45}$. Here, we report that depletion of SETD2 leads to an enrichment of R-loops at telomeres, which in turn facilitates the ALT pathway in the absence of ATRX. This raises the interesting question as to how SETD2 normally modulates the formation of telomeric R-loops? Histone $\mathrm{H} 3 \mathrm{~K} 36 \mathrm{me} 3$ is known to recruit a variety of chromatin associated proteins, many with defined roles in reshaping chromatin accessibility following transcription, including MRG15 (a component of the Rpd3S/Sin3S histone deacetylase complex) ${ }^{60}$, SPT16 (a component of the FACT complex) ${ }^{61}$ and PHF1/19 (a component of the polycomb repressive complex 2 (PRC2)) ${ }^{62,63}$. One possibility therefore is that a more open telomeric chromatin structure upon SETD2 loss is more permissive to the formation of R-loop and/or G-loop structures. In line with this hypothesis,

393 loss of FACT has previously been linked to increases in R-loop formation ${ }^{64}$. Histone H3 K36me3 has also been shown to recruit the DNA methyltransferase DNMT3b, loss of which has been shown to induce hypomethylation at sub-telomeres and the accumulation of telomeric R-loops in patients with ICF (Immunodeficiency, Centromeric instability and Facial

397 anomalies) syndrome ${ }^{65}$. Taken together, the data we present here suggests that it is the combination of increased telomeric R-loops upon SETD2 disruption, in concert with ATRX loss, that triggers the ALT pathway in this cohort of paediatric HGG. Mutations in histone H3.3 at glycine 34 to arginine or valine (G34R/V) are also highly co402 incident with ATRX loss in HGG and tend to be mutually exclusive with SETD2 mutations ${ }^{16,45}$. 403 Indeed, these mutations are thought to impede the ability of SETD2 to methylate the H3 tail, 
suggesting that in all likelihood the mechanism of ALT induction in tumours with this mutational signature phenocopies that of a SETD2 mutant, with the accumulation of

406 telomeric R-loops. H3.3 G34R/V has, however, only been shown to obstruct SETD2 activity in

407 cis ${ }^{66,67}$ and this conjecture is therefore dependent on the mutant histone being incorporated

408 into telomeric chromatin. In support of this possibility, recent work has shown that in the 409 absence ATRX/DAXX, the histone H3.3 chaperone HIRA is enriched at ALT telomeres as an 410 adaptive response to allow the deposition of histone $\mathrm{H} 3.3$ at telomeres ${ }^{68}$. A second mutation

411 in histone $\mathrm{H} 3.3$ at lysine 27 to methionine (K27M) is also highly coincident with ATRX loss and

412 inhibits the catalytic activity of PRC2 $16,45,67$. It will therefore be of interest to ascertain 413 whether the inhibition of PRC2 also elicits ALT markers when combined with ATRX/DAXX loss 414 and, if so, whether loss of PRC2 function leads to the accumulation of aberrant secondary 415 structures on telomeric DNA.

417 ATRX has previously been implicated with multiple roles at the replication fork which could explain the data we present here, including roles in both the protection of stalled forks from

419 nucleolytic degradation ${ }^{28}$ and the restart of stalled replication forks ${ }^{29,32}$. Of note, ATRX has been proposed to interact with factors known to modulate both fork restart and protection.

421 ATRX has been shown to interact and cooperate with FANCD2 to recruit CtIP to stalled replication forks and promote MRE11 dependent fork restart. ATRX itself has also been shown to interact with components of the MRN complex, raising the intriguing possibility that ATRX constitutes a component of a fork restart complex ${ }^{20,29,32}$. Understanding how these interactions are regulated will likely give important insights as to how ATRX facilitates progressive DNA replication through genotoxic lesions and/or aberrant DNA secondary structures. 
428 A second implication of the data presented here is that ATRX/DAXX may have a direct role in

429 the clearing or resolution of structures or genotoxic lesions on telomeric DNA. Of note, ATRX

430 possesses a translocase activity ${ }^{69}$ which could potentially resolve R-loops via branch

431 migration. Indeed, loss of ATRX has been shown to lead to an increase in telomeric R-loops at

432 a highly transcribed ectopic telomeric repeat ${ }^{27}$, consistent with a role for ATRX in clearing R-

433 loops once they have been triggered. R-loops have also been suggested to be excised by the

434 nucleotide excision repair (NER) nucleases XPG and XPF, resulting in the formation of a single

435 strand break, which could then ultimately trigger fork collapse in S-phase ${ }^{70}$. Interestingly,

436 histone H3.3 deficient chicken DT40 cells have recently been shown to exhibit a likely defect

437 in NER ${ }^{71}$, raising the interesting possibility that ATRX/DAXX may be the histone H3.3

438 chaperone facilitating the completion of this process. In line with this notion, the nicking of

439 telomeric DNA to form single stand breaks using the CRISPR-Cas9 system has been shown to

440 induce the formation of C-circles in ATRX proficient cells ${ }^{7}$.

441

442 Finally, we report that the KO of ATRX in HeLa LT cells is associated with a marked loss of

443 telomere sister chromatid cohesion, consistent with a recent report in mouse cells, published

444 during the preparation of this manuscript ${ }^{49}$. How ATRX normally maintains telomere

445 cohesion remains an interesting question, nonetheless this observation likely accounts for the

446 net gain in telomere length observed as a result of the BIR-like pathway in ALT cancer cells by

447 facilitating the use of an out of register telomere template. Taken together, work presented

448 here suggests that ATRX has a multi-faceted role in the suppression of the ALT pathway,

449 accounting for its role as a near universal tumour suppressor in these cancers. Firstly, ATRX

450 protects replication forks from collapse in the presence of telomeric lesions including R-loop

451 structures. Secondly, ATRX maintains cohesion between sister telomeres, ensuring the 
faithful use of BIR without aberrant increases in telomere length. Finally, we provide evidence

that the induction of ALT requires two independent cellular events; the loss of ATRX/DAXX in conjunction with the loss of another factor or factors that lead to the formation of lesions or structures at telomeres, as exemplified here by SETD2 (Figure 7C).

\section{ACKNOWLEDGEMENTS}

We thank Prof. Amato Giaccia and Dr. Andrew Blackford for helpful discussions. HeLa LT cells were a kind gift from Dr. Roderick O'Sullivan. We acknowledge Kevin Clark in the MRC Weatherall Institute of Molecular Medicine (WIMM) Flow Cytometry Facility for providing cell-sorting services and technical expertise; Dominic Waithe and Christoffer Lagerholm in the Wolfson Imaging Centre for help with microscopy and data analysis; Philip Hublitz in MRC WIMM Genome Engineering Facility for preparation of RNase H overexpression vectors; Ryan Beveridge in the MRC WIMM Virus Screening Facility for help in generation of lentiviral preparations. These facilities are supported by the MRC Molecular Hematology Unit (MC_UU_12009), the MRC Human Immunology Unit (MC_UU_12010), the Wolfson Foundation (grant 18272), the Wellcome Trust (Micron107457/Z/15Z), the National Institute for Health Research Oxford Biomedical Research Centre (IS-BRC-1215-20008), the Cancer Research UK (CRUK) Oxford Centre, the KayKendall Leukemia Fund (KKL1057), the John Fell Fund (131/030 and 101/517), the EPA fund (CF182 and CF170) and the MRC WIMM Strategic Alliance (G0902418 andMC_UU_12025). The Clynes laboratory is supported through a Children with Cancer UK Paul O’ Gorman Career Development Fellowship (15-202). T.K. and T.G. are supported through MRC Studentships. S.S. is funded through CRUK OIRO 
475 (C5255/A23755). S.K. is supported through a Kwanjeong Educational Foundation (KEF) PhD 476 scholarship. S.C. is funded through MRC OIRO (MC_UU_00001).

477

\section{AUTHOR CONTRIBUTIONS}

479

480 This project was conceived and supervised by D.C. with support from R.J.G and T.H. T.G., T.K., 481 S.S. and S.C. performed the majority of the experiments. S.K. performed all cell proliferation 482 assays. D.C. wrote the manuscript. T.K. and T.G. prepared the figures. R.J.G, T.H, T.G., and T.K. 483 critically reviewed the manuscript.

484

485 DECLARATION OF INTERESTS

486

487 The authors declare no competing interests.

488

489 
Cell lines and cell culture conditions

493

494 Cell lines used are listed in Supplementary Table 2. All cell lines were obtained from ATCC with the exception of HeLa LT which was a kind gift from Roderick O'Sullivan ${ }^{34}$ and HeLa ST

496 (Hela H3.3-SNAP) were a kind gift from Genevieve Almouzni ${ }^{72}$. All cells were cultured in 497 standard Dulbecco's Modified Eagle's Medium (DMEM) media supplemented with $10 \%$ foetal 498 calf serum, 1\% L-glutamine and 1\% PenStrep (Gibco). U-2 OS ATRX cells were cultured in the presence of $0.4 \mu \mathrm{g} / \mathrm{mL}$ puromycin and $175 \mu \mathrm{g} / \mathrm{mL}$ neomycin. Ectopic ATRX expression was driven through the addition of $0.4 \mu \mathrm{g} / \mathrm{mL}$ doxycycline.

501

CRISPR-Cas9 KO of ATRX was performed using a modified pSpCas9(bb)-2A-GFP tagged Cas9 vector containing sgRNAs targeting exon 16 of ATRX (sgRNA sequence - TOP: 5' caccGTCCAATAACAACCA^AGT 3’, BOTTOM: 5’ aaaACT^^TGGTTGTTATTGGAC 3’) with an expected cut site at lysine 1536. Cells were sorted on a BD FACSAria Fusion cell sorter 24 hours after transfection into single wells and grown into clones. KO was determined by western blotting. DAXX KOs were performed using a commercially available DAXX Cas9 plasmid (Santa Cruz, sc-400686-KO-2) and were FACs sorted based on GFP expression. SETD2

511 Cas9 KO was performed as described in ${ }^{73}$. Transfected cells were selected using $0.4 \mu \mathrm{g} / \mathrm{mL}$ 512 puromycin and then sorted into single wells to obtain clones. Combinatorial KOs were made 513 through sequential KO of genes. 
516 Cells were treated with these genotoxic agents at the following doses for 48 hours prior to 517 downstream analysis with the ALT assays used in this study: PDS at $5 \mu \mathrm{M}, \mathrm{CPT}$ at $50 \mathrm{nM}, \mathrm{APH}$ 518 at $0.2 \mu \mathrm{M}$ and ETO at $0.5 \mu \mathrm{M}$.

SiRNA Knockdown

Mus81 siRNA experiments were performed using the commercially available ON-TARGETplus Mus81 siRNA SmartPool (Dharmacon, L-016143-01-0005). Cells were reverse transfected

524 when seeding cells to wells or coverslips using Lipofectamine RNAiMAX, to a final concentration of $5 \mathrm{pmol}$ following the manufacturer's instructions. 48 hours after transfection well were forward transfected again to ensure consistent knockdown. For Mus81 experiments, 72 hours after transfection cells were treated with $5 \mu \mathrm{M}$ PDS and incubated for a further 24 hours before harvesting.

ImmunoFISH

ImmunoFISH experiments were performed with cells seeded onto $13 \mathrm{~mm} \# 00$ thickness coverslips. Briefly, cells were pre-permeabilised with $0.5 \%$ Triton-X100 for 1 minute on ice then fixed in $4 \%$ paraformaldehyde for 20 minutes. Fixed coverslips were washed in PBS then permeabilised on ice for 6 minutes with $0.5 \%$ Triton-X100. Coverslips were blocked in blocking buffer (2\% BSA, 0.1\% Tween-20 in PBS) for 1 hour and then incubated with the appropriate

537 primary antibody in blocking buffer. Following primary antibody staining, coverslips were 
washed in PBST (0.5\% Tween-20 in PBS) and stained with an appropriate Alexa fluorophoreconjugated secondary antibody. Following further washing, coverslips were post-fixed with

$5404 \%$ PFA for 20 minutes and then a Cy3-[CCCTTA $]_{5}$ probe was hybridised onto the coverslip in 541 hybridisation buffer (25\% formamide, 2 XSSC, $200 \mathrm{ng} / \mu \mathrm{L}$ Salmon sperm, $5 x$ Denhardts solution,

$54250 \mathrm{mM}$ phosphase buffer, $1 \mathrm{mM}$ EDTA in water) overnight at $37^{\circ} \mathrm{C}$ in a humidified chamber. In

543 instances where denatured telomere signals were required, coverslips were incubated in 3.5

$544 \mathrm{~N} \mathrm{HCl}$ for 13 minutes, then immediately washed with ice cold PBS prior to hybridisation with

545 telomere probes. For analysis of single stranded telomeric DNA the denaturation step was

546 omitted. Coverslips were then washed in 2x SSC before being mounted in VectaShield containing DAPI. Coverslips were then visualised using a DeltaVision widefield microscope.

C-circle Assay

550

551

DNA was extracted from approximately $1 \times 10^{6}$ cells using the QIAGEN Core B kit. Extracted

DNA was resuspended in $20 \mathrm{mM}$ Tris- $\mathrm{HCl}$. DNA was quantified and then $30 \mathrm{ng}$ of genomic DNA was amplified in a PCR reaction containing $\$ 29$ polymerase, $1 \%$ Tween- $20,200 \mu \mathrm{g} / \mathrm{mL}$ BSA and dTTP, dGTP, and dATP, but lacking dCTP for 8 hours at $30^{\circ} \mathrm{C}$ followed by 20 minutes at

$65^{\circ} \mathrm{C}$. PCR reactions were run with and without $\phi 29$ polymerase to ensure the signal was specific for rolling-circle amplification products. Amplified samples were then blotted onto Zeta-Probe membrane (Bio-Rad) using a slot blotter.

Following fixing of DNA to the membrane with a UVA Stratalinker 2400, membranes were soaked in PerfectHyb Plus (Sigma Aldrich) for 20 minutes at room temperature. A 3' Digitonin

561 (DIG) tagged $[\text { CCCATT }]_{5}$ oligonucleotide was then diluted in PerfectHyb to a final 
concentration of $40 \mathrm{nM}$ in $20 \mathrm{~mL}$ hybridisation buffer, and was allowed to hybridise with the membrane in a rolling incubator for 2 hours at $37^{\circ} \mathrm{C}$. Following hybridisation, membranes were briefly washed twice with wash buffer (0.1M Maleic acid, $3 \mathrm{M} \mathrm{NaCl} ; 0.1 \%$ Tween 20 adjusted to $\mathrm{pH}$ 7.5), before blocking for 30 minutes and probing with a DIG antibody for 30

566 minutes. Finally, membranes were vigorously washed 3 times with wash buffer before placing

567 into a cassette with CDP-Star solution. Blots were then developed onto Amersham Hyperfilm

568 ECL film.

Terminal Restriction Fragment (TRF) assay

572 Terminal restriction fragment assays were performed by first extracting genomic DNA as

573 described above with the QIAGEN Core B kit. Extracted DNA was quantified and at least $2 \mu \mathrm{g}$

574 of genomic DNA was digested overnight at $37^{\circ} \mathrm{C}$ with Hinfl and Rsal restriction endonucleases.

575 Following digestion, samples were run on $0.8 \%$ agarose gels in $1 \times$ TAE at $60 \mathrm{~V}$ overnight. Gels were denatured using $1 \mathrm{M} \mathrm{NaOH}$ and then neutralised and blotted onto Zeta-Probe

577 membrane by upward capillary transfer. Blots were then probed with a DIG-tagged telomere probe as described above. The blots were analysed and quantified using TeloMetric software 74.

Monochrome multiplex $q P C R(m m-q P C R)$

MM-qPCR was carried out as described in ${ }^{75}$ with some alterations. Genomic DNA samples were diluted to $5 \mathrm{ng} / \mu \mathrm{L}$. Primer sets are listed in Supplementary Table 3. Five concentrations of reference genomic DNA purified from HeLa LT were prepared by 3-fold serial dilution (from 
$150 \mathrm{ng}$ to $1.85 \mathrm{ng}$ ) to generate standard curves for relative quantitation of $\mathrm{T} / \mathrm{S}$ ratios. For each

587 sample, $20 \mathrm{ng}$ of genomic DNA was mixed with $0.75 \times$ PowerUp SYBR Green Master Mix

588 (Thermo Scientific), the primers (300 nM), and water to a final volume of $20 \mu \mathrm{L}$ per well and analysed using a Thermo Fisher QuantStudio 3 qPCR machine with the following cycle

590 conditions: denaturation for $15 \mathrm{~min}$ at $95{ }^{\circ} \mathrm{C}$, followed by two cycles of $15 \mathrm{~s}$ at $94{ }^{\circ} \mathrm{C} / 15 \mathrm{~s}$ at $49^{\circ} \mathrm{C}$ and 32 cycles of $15 \mathrm{~s}$ at $94^{\circ} \mathrm{C} / 10 \mathrm{~s}$ at $62^{\circ} \mathrm{C} / 15 \mathrm{~s}$ at $74{ }^{\circ} \mathrm{C}$ with signal acquisition and $10 \mathrm{~s}$ at $84^{\circ} \mathrm{C} / 15 \mathrm{~s}$ at $88^{\circ} \mathrm{C}$ with signal acquisition. Samples were run in triplicate, and analysis was repeated six times using independent runs.

Cohesion FISH

Cohesion FISH was performed using commercially available FISH probe set from Cytocell 598 designed to detect DLEU7 deletion (Cytocell, LPH-043-S) following the manufacturers protocol. Coverslips were then visualised using a DeltaVision widefield microscope.

600

601

S9.6 DNA-RNA Immunoprecipitation (DRIP)

602

R-loop DRIP experiments were performed using the $\$ 9.6$ antibody as described in ${ }^{27}$. Eluted

604 DNA was analysed by slot blotting and probing using a DIG-tagged telomere probe as described above.

606

607

608

609 
612 HeLa-LT cells were seeded on opaque 96 -well plate at $100-500$ cells in $100 \mu$ l of culture media

613 per well. After 6-24 hours, an equal volume of the drug-containing media was added at the 614 desired final concentrations. HU and APH treatment was performed for 72 hours and PDS

615 treatment was performed for 7 days. The cell proliferation was assessed using CellTiter-Glo 6162.0 Reagent (ProMega) according to the manufacturer's instructions. Briefly, the plate and 617 the reagent were equilibrated at room temperature for 5 minutes, the media was removed 618 from the plate, and $100 \mu$ of the diluted reagent (1:5 in PBS) was added to each well. After 61910 minutes, the plate was read via the ProMega GloMax Luminometer to assess the quantity 620 of metabolically active cells. The IC50 values were derived by fitting the dose-response data 621 into the curves via GraphPad Prism v8.0.

Triptolide Treatment and Cell Cycle Analysis by PI Staining

ATRX/SETD2 double KO cells were treated with increasing doses of the transcription inhibitor triptolide ( $25 \mathrm{nM}, 50 \mathrm{nM}$ and $100 \mathrm{nM}$ ) for 24 hours. gDNA was then extracted and the C-circle assay was performed as described above. To ensure cells were still progressing through the cell cycle, PI staining was utilised. Briefly, cells were harvested, washed in 1x PBS and fixed in $70 \%$ ethanol on ice for 30 minutes. Cells were washed again in PBS, treated with $100 \mu \mathrm{g} / \mathrm{ml}$ RNase $A$ and then incubated with $50 \mu \mathrm{g} / \mathrm{ml}$ propidium iodide solution for 10 minutes at room temperature. Samples were then analysed by flow cytometry to estimate the percentage of cells in $\mathrm{G} 1, \mathrm{~S}$ and $\mathrm{G} 2$ phases of the cell cycle. 
636 Lentivirus-based constructs for overexpression of wildtype RNase $\mathrm{H}$ and the catalytically dead

637 D210N mutant in HeLa cells were generated using InFusion cloning to insert the NLS-RNaseH638 V5 sequences from plasmids ppCAG_RNaseH1_WT (Addgene \#111906) and 639 ppCAG_RNaseH1_D210N (Addgene \#111904) into the pLeGO-C2 backbone (Addgene 640 \#27339). The ATRX/SETD2 double KO cells were transduced with the lentivirus and left for 14 641 days before DNA was harvested and C-circle analysis was carried out as described above.

642 Efficient overexpression was assessed by Western blot.

643

644 Quantification and Statistical Analysis

645

646 Each experiment was repeated at least twice, with representative results shown. Statistical 647 analysis was done using GraphPad Prism 9 (GraphPad Software Inc.). Unpaired t tests were 648 used to compare two groups. One-way ANOVA was used to compare more than two groups. 649 Sample sizes and $p$-values are shown in the figure legends. In all cases ns indicates $p>0.05$. 650 
653 1. de Lange, T. Shelterin-Mediated Telomere Protection. (2018) doi:10.1146/annurev$654 \quad$ genet-032918.

655 2. Heaphy, C. M. et al. Prevalence of the alternative lengthening of telomeres telomere maintenance mechanism in human cancer subtypes. American Journal of Pathology $179,1608-1615$ (2011).

3. Dilley, R. L. et al. Break-induced telomere synthesis underlies alternative telomere maintenance. Nature 539, 54-58 (2016).

4. Min, J., Wright, W. E. \& Shay, J. W. Alternative Lengthening of Telomeres Mediated by doi:10.1128/MCB.

5. Roumelioti, F. et al. Alternative lengthening of human telomeres is a conservative DNA replication process with features of break-induced replication . EMBO reports 17, 1731-1737 (2016).

6. Cho, N. W., Dilley, R. L., Lampson, M. A. \& Greenberg, R. A. Interchromosomal homology searches drive directional ALT telomere movement and synapsis. Cell 159, 108-121 (2014).

7. Zhang, T. et al. Strand break-induced replication fork collapse leads to C-circles, Coverhangs and telomeric recombination. PLoS Genetics 15, (2019).

8. Sfeir, A. et al. Mammalian Telomeres Resemble Fragile Sites and Require TRF1 for Efficient Replication. Cell 138, 90-103 (2009).

9. Aguilera, A. \& García-Muse, T. R Loops: From Transcription Byproducts to Threats to Genome Stability. Molecular Cell vol. 46 115-124 (2012). 
675 10. Silva, B. et al. FANCM limits ALT activity by restricting telomeric replication stress induced by deregulated BLM and R-loops. Nature Communications 10, (2019).

11. Pan, X. et al. FANCM suppresses DNA replication stress at ALT telomeres by disrupting TERRA R-loops. Scientific Reports 9, (2019).

12. Lu, R. et al. The FANCM-BLM-TOP3A-RMI complex suppresses alternative lengthening of telomeres (ALT). Nature Communications 10, (2019).

13. Arora, R. et al. RNaseH1 regulates TERRA-telomeric DNA hybrids and telomere 682 maintenance in ALT tumour cells. Nature Communications 5, (2014).

14. Pan, X. et al. FANCM, BRCA1, and BLM cooperatively resolve the replication stress at the ALT telomeres. Proceedings of the National Academy of Sciences of the United States of America 114, E5940-E5949 (2017).

15. Lovejoy, C. A. et al. Loss of ATRX, genome instability, and an altered DNA damage response are hallmarks of the alternative lengthening of Telomeres pathway. PLoS Genetics 8, (2012).

16. Schwartzentruber, J. et al. Driver mutations in histone H3.3 and chromatin remodelling genes in paediatric glioblastoma. Nature 482, 226-231 (2012).

691 17. Napier, C. E. et al. ATRX represses alternative lengthening of telomeres. Oncotarget vol. 6 www.impactjournals.com/oncotarget/ (2015).

18. Clynes, D. et al. Suppression of the alternative lengthening of telomere pathway by the chromatin remodelling factor ATRX. Nature Communications 6, (2015).

19. Eid, R. et al. Genetic Inactivation of ATRX Leads to a Decrease in the Amount of Telomeric Cohesin and Level of Telomere Transcription in Human Glioma Cells . Molecular and Cellular Biology 35, 2818-2830 (2015). 
698 20. Clynes, D. et al. ATRX dysfunction induces replication defects in primary mouse cells. PLOS ONE 9, (2014).

21. Brosnan-Cashman, J. A. et al. ATRX loss induces multiple hallmarks of the alternative lengthening of telomeres (ALT) phenotype in human glioma cell lines in a cell linespecific manner. PLOS ONE 13, (2018).

22. Clynes, D., Higgs, D. R. \& Gibbons, R. J. The chromatin remodeller ATRX: A repeat offender in human disease. Trends in Biochemical Sciences vol. 38 461-466 (2013).

23. Drané, P., Ouararhni, K., Depaux, A., Shuaib, M. \& Hamiche, A. The death-associated protein DAXX is a novel histone chaperone involved in the replication-independent deposition of H3.3. Genes and Development 24, 1253-1265 (2010).

24. Goldberg, A. D. et al. Distinct Factors Control Histone Variant H3.3 Localization at Specific Genomic Regions. Cell 140, 678-691 (2010).

25. Wong, L. H. et al. ATRX interacts with H3.3 in maintaining telomere structural integrity in pluripotent embryonic stem cells. Genome Research 20, 351-360 (2010).

26. Wang, Y. et al. G-quadruplex DNA drives genomic instability and represents a targetable molecular abnormality in ATRX-deficient malignant glioma. G-quadruplex DNA drives genomic instability and represents a targetable molecular abnormality in ATRX-deficient malignant glioma 347542 (2018) doi:10.1101/347542.

27. Nguyen, D. T. et al. The chromatin remodelling factor ATRX suppresses R-loops in transcribed telomeric repeats . EMBO reports 18, 914-928 (2017).

28. Huh, M. S. et al. Stalled replication forks within heterochromatin require ATRX for protection. Cell Death and Disease 7, (2016). 
29. Leung, J. W. C. et al. Alpha thalassemia/mental retardation syndrome X-linked gene product ATRX is required for proper replication restart and cellular resistance to replication stress. Journal of Biological Chemistry 288, 6342-6350 (2013).

30. Watson, L. A. et al. Atrx deficiency induces telomere dysfunction, endocrine defects, and reduced life span. Journal of Clinical Investigation 123, 2049-2063 (2013).

31. Juhász, S., Elbakry, A., Mathes, A. \& Löbrich, M. ATRX Promotes DNA Repair Synthesis and Sister Chromatid Exchange during Homologous Recombination. Molecular Cell 71, 11-24.e7 (2018).

32. Raghunandan, M. et al. Functional cross talk between the Fanconi anemia and ATRX/DAXX histone chaperone pathways promotes replication fork recovery. Human molecular genetics 29, 1083-1095 (2020).

33. Koschmann, C. et al. C A N C E R ATRX loss promotes tumor growth and impairs nonhomologous end joining DNA repair in glioma. Science Translational Medicine 8, undefined (2016).

34. O'Sullivan, R. J. et al. Rapid induction of alternative lengthening of telomeres by depletion of the histone chaperone ASF1. Nature Structural and Molecular Biology 21, 167-174 (2014).

35. Spiegel, J., Adhikari, S. \& Balasubramanian, S. The Structure and Function of DNA GQuadruplexes. Trends in Chemistry vol. 2 123-136 (2020).

36. Law, M. J. et al. ATR-X syndrome protein targets tandem repeats and influences allelespecific expression in a size-dependent manner. Cell 143, 367-378 (2010).

37. Loe, T. K. et al. Telomere length heterogeneity in ALT cells is maintained by PMLdependent localization of the BTR complex to telomeres. Genes and Development 34, 650-662 (2020). 
38. Bryan, T. M., Englezou, A., Gupta, J., Bacchetti, S. \& Reddel, R. R. Telomere elongation in immortal human cells without detectable telomerase activity. EMBO Journal 14, 4240-4248 (1995).

39. Bakkenist, C. J. \& Kastan, M. B. DNA damage activates ATM through intermolecular autophosphorylation and dimer dissociation. www.nature.com/nature (2003).

40. Pepe, A. \& West, S. C. MUS81-EME2 promotes replication fork restart. Cell Reports 7, (2014).

41. Zeng, S. et al. Telomere recombination requires the MUS81 endonuclease. Nature Cell Biology 11, 616-623 (2009).

42. Zellweger, R. et al. Rad51-mediated replication fork reversal is a global response to genotoxic treatments in human cells. Journal of Cell Biology 208, 563-579 (2015).

43. Ray Chaudhuri, A. et al. Topoisomerase $\mathrm{i}$ poisoning results in PARP-mediated replication fork reversal. Nature Structural and Molecular Biology 19, 417-423 (2012).

44. Udugama, M. et al. Ribosomal DNA copy loss and repeat instability in ATRX-mutated cancers. Proceedings of the National Academy of Sciences of the United States of America 115, 4737-4742 (2018).

45. Fontebasso, A. M. et al. Mutations in SETD2 and genes affecting histone H3K36 methylation target hemispheric high-grade gliomas. Acta Neuropathologica 125, 659669 (2013).

46. Kanu, N. et al. SETD2 loss-of-function promotes renal cancer branched evolution through replication stress and impaired DNA repair. Oncogene 34, 5699-5708 (2015).

47. Pan, X. et al. FANCM, BRCA1, and BLM cooperatively resolve the replication stress at the ALT telomeres. Proceedings of the National Academy of Sciences of the United States of America 114, E5940-E5949 (2017). 
48. Yang, Z., Takai, K. K., Lovejoy, C. A. \& de Lange, T. Break-induced replication promotes fragile telomere formation. Genes \& Development (2020) doi:10.1101/gad.328575.119.

49. Lovejoy, C. A., Takai, K., Huh, M. S., Picketts, D. J. \& de Lange, T. ATRX affects the repair of telomeric DSBs by promoting cohesion and a DAXX-dependent activity. PLoS biology 18, e3000594 (2020).

50. Graham, M. K. et al. Functional loss of ATRX and TERC activates Alternative Lengthening of Telomeres (ALT) in LAPC4 prostate cancer cells. Molecular Cancer Research 17, 2480-2491 (2019).

51. Huh, M. S. et al. Compromised genomic integrity impedes muscle growth after Atrx inactivation. Journal of Clinical Investigation 122, 4412-4423 (2012).

52. Taylor, M. R. G. \& Yeeles, J. T. P. The Initial Response of a Eukaryotic Replisome to DNA Damage. Molecular Cell 70, 1067-1080.e12 (2018).

53. de Magis, A. et al. DNA damage and genome instability by G-quadruplex ligands are mediated by $\mathrm{R}$ loops in human cancer cells. Proceedings of the National Academy of Sciences of the United States of America 116, 816-825 (2019).

54. Olivieri, M. et al. A Genetic Map of the Response to DNA Damage in Human Cells. Cell 182, 481-496.e21 (2020).

55. Tammaro, M., Barr, P., Ricci, B. \& Yan, H. Replication-dependent and transcriptiondependent mechanisms of DNA double-strand break induction by the topoisomerase 2-targeting drug etoposide. PLOS ONE 8, (2013).

56. Sordet, O. et al. Ataxia telangiectasia mutated activation by transcription- and topoisomerase I-induced DNA double-strand breaks. EMBO Reports 10, 887-893 (2009). 
57. Marinello, J., Chillemi, G., Bueno, S., Manzo, S. G. \& Capranico, G. Antisense transcripts enhanced by camptothecin at divergent CpG-island promoters associated with bursts of topoisomerase I-DNA cleavage complex and R-loop formation. Nucleic Acids Research 41, 10110-10123 (2013).

58. O'Sullivan, R. J. et al. Rapid induction of alternative lengthening of telomeres by depletion of the histone chaperone ASF1. Nature Structural and Molecular Biology 21, 167-174 (2014).

59. Kang, M. R., Muller, M. T. \& Chung, I. K. Telomeric DNA damage by topoisomerase I: A possible mechanism for cell killing by camptothecin. Journal of Biological Chemistry 279, 12535-12541 (2004).

60. Kumar, G. S. et al. Sequence requirements for combinatorial recognition of histone H3 by the MRG15 and Pf1 subunits of the Rpd3S/Sin3S corepressor complex. Journal of Molecular Biology 422, 519-531 (2012).

61. Carvalho, S. et al. Histone methyltransferase SETD2 coordinates FACT recruitment with nucleosome dynamics during transcription. Nucleic Acids Research 41, 2881-2893 (2013).

62. Ballaré, C. et al. Phf19 links methylated Lys36 of histone H3 to regulation of Polycomb activity. Nature Structural and Molecular Biology 19, 1257-1265 (2012).

63. Cai, L. et al. An H3K36 Methylation-Engaging Tudor Motif of Polycomb-like Proteins Mediates PRC2 Complex Targeting. Molecular Cell 49, 571-582 (2013).

64. Herrera-Moyano, E., Mergui, X., García-Rubio, M. L., Barroso, S. \& Aguilera, A. The yeast and human FACT chromatinreorganizing complexes solve R-loopmediated transcription-replication conflicts. Genes and Development 28, 735-748 (2014). 
815 65. Sagie, S. et al. Telomeres in ICF syndrome cells are vulnerable to DNA damage due to elevated DNA:RNA hybrids. Nature Communications 8, (2017).

817 66. Weinberg, D. N., Allis, C. D. \& Lu, C. Oncogenic mechanisms of histone H3 mutations. Cold Spring Harbor Perspectives in Medicine vol. 7 (2017).

819 67. Lewis, P. W. et al. Inhibition of PRC2 activity by a gain-of-function H3 mutation found in pediatric glioblastoma. Science 340, 857-861 (2013).

68. Hoang, S. M. et al. Regulation of ALT-associated homology-directed repair by polyADP-ribosylation. Nature Structural and Molecular Biology (2020) doi:10.1038/s41594-020-0512-7.

69. Xue, Y. et al. The ATRX syndrome protein forms a chromatin-remodeling complex with Daxx and localizes in promyelocytic leukemia nuclear bodies. vol. 100 www.pnas.orgcgidoi10.1073pnas.1937626100 (2003).

70. Sollier, J. et al. Transcription-Coupled Nucleotide Excision Repair Factors Promote RLoop-Induced Genome Instability. Molecular Cell 56, 777-785 (2014).

71. Frey, A., Listovsky, T., Guilbaud, G., Sarkies, P. \& Sale, J. E. Histone H3.3 is required to maintain replication fork progression after UV damage. Current Biology 24, 2195-2201 (2014).

72. Adam, S., Polo, S. E. \& Almouzni, G. XTranscription recovery after DNA damage requires chromatin priming by the H3.3 histone chaperone HIRA. Cell 155, 94 (2013).

834 73. Pfister, S. X. et al. Inhibiting WEE1 Selectively Kills Histone H3K36me3-Deficient Cancers by dNTP Starvation. Cancer Cell 28, 557-568 (2015).

74. Grant, J. D. et al. Telometric: A Tool Providing Simplified, Reproducible Measurements of Telomeric DNA from Constant Field Agarose Gels. BioTechniques 31, 1314-1318 (2001). 
839 75. Cawthon, R. M. Telomere length measurement by a novel monochrome multiplex 840 quantitative PCR method. Nucleic Acids Research 37, (2009).

841

842 
845 Figure 1. Loss of ATRX alone is insufficient to trigger the ALT pathway. A) Immunoblot confirming CRISPR-Cas9 mediated KO of ATRX in two independent clones of HeLa LT. B-C) C-

847 circle blot and quantification showing that deletion of ATRX in the HeLa LT cell line is 848 insufficient to trigger the accumulation of C-circles, $n=3$. - Phi 29 is a negative control 849 performed without the addition of the phi29 polymerase. U-2 OS is included as an ALT850 positive positive control. D-E) ImmunoFISH detection of ALT associated PML Nuclear Bodies

851 (APBs) both in the presence and absence of ATRX, showing no induction of APB formation 852 upon ATRX loss, >200 nuclei analysed across 3 biological replicates. F) Terminal Restriction Fragment (TRF) analysis of telomere length showing no overt changes in telomere length or heterogeneity upon ATRX deletion. In all panels, ns signifies $p>0.05$, determined by one-way ANOVA.

856

Figure 2. Treatment with PDS in combination with ATRX loss triggers ALT markers. A-B) C-

858 circle blot and quantification showing C-circle accumulation specifically in ATRX KO clones following $48 \mathrm{hrs}$ treatment with $5 \mu \mathrm{M}$ PDS, $\mathrm{n}=3 .{ }^{* * * *} \mathrm{p}<0.0001$ determined by one-way

860 ANOVA. C-D) Representative images and quantification of APB induction in HeLa LT ATRX KO 861 clones upon 48 hrs treatment with $5 \mu \mathrm{M}$ PDS, $>200$ nuclei analysed across 3 biological 862 replicates. ${ }^{* *} \mathrm{p}<0.001, * * * * \mathrm{p}<0.0001$, determined by one-way ANOVA. E-F) Representative images and quantification of RPA ssTel in HeLa LT ATRX KO upon treatment with $48 \mathrm{hrs} 5 \mu \mathrm{M}$ PDS, $>150$ nuclei analysed across 3 biological replicates. $* * p<0.001, * * * * p<0.0001$,

865 determined by one-way ANOVA. G) TRF analysis showing treatment with $5 \mu \mathrm{M}$ PDS for 48 hrs 866 increased telomere length heterogeneity specifically upon depletion of ATRX. Telomere 
heterogeneity was measured using Telometric software, $n=2 .{ }^{*} p<0.05$, determined by oneway ANOVA.

869

Figure 3. PDS induced ALT markers require replication fork collapse. A-B) Representative

figure and quantification of pATM induction at telomeres upon PDS treatment. >150 nuclei analysed across 3 biological replicates. ${ }^{* *} p<0.001,{ }^{* * *} p<0.0001,{ }^{* * * * p}<0.0001$, determined by one-way ANOVA. C) Immunoblot showing siRNA mediated knockdown of Mus81 in ATRX WT and ATRX KO cells. D) C-circle quantification showing induction of C-circles upon ATRX loss and PDS treatment is dependent on Mus81, $\mathrm{n}=3 .{ }^{*} \mathrm{p}<0.05,{ }^{*} \mathrm{p}<0.001$, determined by one-way ANOVA. E-F) Representative images and quantification from ImmunoFISH data showing the induction of RPA ssTel foci upon ATRX loss and PDS treatment is dependent on Mus81, $>150$ nuclei analysed across 3 biological replicates. $* * * * p<0.0001$, determined by one-way ANOVA.

880 markers for HeLa LT ATRX KO cells treated with low dose CPT, ETO and APH. A) C-circle blot and quantification, $\mathrm{n}=3 .{ }^{*} \mathrm{p}<0.05, * * \mathrm{p}<0.001, * * * \mathrm{p}<0.0001, * * * * \mathrm{p}<0.0001$, determined by one-way ANOVA. B) Representative ImmunoFISH to detect APBs following treatment of ATRX KO cells with CPT, APH and ETO, >200 nuclei analysed across 3 biological replicates. C) Quantification of APBs in treated HeLa LT cells. ${ }^{* * * * p}<0.0001$, determined by one-way ANOVA. D) Representative RPA ssTel immunoFISH images in HeLa LT ATRX KO cells treated with the same panel of genotoxic agents. E) Quantification of RPA ssTel foci, $>150$ nuclei analysed across 3 biological replicates. ${ }^{*} p<0.05,{ }^{* *} p<0.001,{ }^{* * * *} p<0.0001$, determined by one-way ANOVA. 
892 Figure 5: ATRX and DAXX act epistatically in the suppression of induced ALT. A) Immunoblot 893 of CRISPR-Cas9 mediated DAXX knockouts in HeLa LT ATRX WT and HeLa LT ATRX 1 cell lines.

894 B-C) C-circle blot and quantification in DAXX KO clones following treatment with PDS and CPT, $895 \mathrm{n}=3 .{ }^{*} \mathrm{p}<0.05, * * \mathrm{p}<0.001, * * * \mathrm{p}<0.0001, * * * * \mathrm{p}<0.0001$, determined by one-way ANOVA.

896 D-E) RPA ssTel representative images and quantification in DAXX KO clones following PDS

897 treatment, $>200$ nuclei analysed across 3 biological replicates. $p<0.0001, * * * * p<0.0001$, 898 determined by one-way ANOVA. F-G) Quantification of APBs following PDS or CPT treatment 899 in ATRX/DAXX KO clones, $>200$ nuclei analysed across 3 biological replicates. ${ }^{* *} p<0.001$, 900 $* * * p<0.0001, * * * * p<0.0001$, determined by one-way ANOVA.

Figure 6. SETD2 loss in combination with ATRX loss triggers markers of the ALT pathway. A) Immunoblot showing loss of H3 K36me3 upon CRISPR-Cas9 mediated deletion of SETD2. B-C) C-circle blot and quantification showing accumulation of C-circles specifically upon codeletion of both ATRX and SETD2 in HeLa LT cells, $n=3 .{ }^{* *} p<0.001, * * * p<0.0001$, determined by one-way ANOVA. D) APB analysis showing accumulation of APBs specifically upon codepletion of ATRX and SETD2, $>100$ nuclei analysed across 3 biological replicates. ${ }^{* *} p<0.001$, 908 determined by one-way ANOVA. E) Quantification of RPA ssTel positive telomeres upon co909 depletion of ATRX and SETD2, >150 nuclei analysed across 2 biological replicates.

$910 \quad 0.0001$, determined by one-way ANOVA. F) Immunoblot showing combined loss of ATRX and

911 SETD2 leads to DNA damage. G) C-circle assay quantification in ATRX/SETD knockout cells

912 treated with triptolide at indicated concentrations, $\mathrm{n}=2 .{ }^{*} \mathrm{p}<0.05,{ }^{*} \mathrm{p}<0.001, * * * \mathrm{p}<0.0001$,

913 determined by one-way ANOVA. H) S9.6 DRIP slot blot and quantification showing increased 914 levels of $\mathrm{R}$ loops at telomeres upon SETD2 loss, $\mathrm{n}=3 .{ }^{* *} \mathrm{p}<0.001$, determined by unpaired 
915 Student's t test. I) Immunoblot showing overexpression of RNase H WT and the catalytically

916 dead D210N mutant in the HeLa LT ATRX/SETD2 1114 days after lentiviral transduction. J)

917 S9.6 DRIP slot blot and quantification showing loss of telomeric R-loops upon ectopic

918 expression of RNase H WT but not the D210N mutant, n=2. K) C-circle blot and quantification

919 of ATRX/SETD2 1 cells overexpressing RNase H WT or the D210N mutant 14 days following

920 transfection, $\mathrm{n}=3 .{ }^{* *} \mathrm{p}<0.001$, determined by unpaired Student's $\mathrm{t}$ test.

921

922

Figure 7. Loss of ATRX induces loss of telomere sister chromatid cohesion. A) FISH of $156 \mathrm{~kb}$

923 region of chromosome 13 covering the D3S319-del genetic marker (red) and 13qter

924 subtelomeric probe (green). Loss of sister chromatid cohesion is observed as a doublet, $>50$

925 nuclei analysed across 2 biological replicates. B) Quantification of doublet formation using the

926 13qter subtelomeric probe. ${ }^{*} p<0.05,{ }^{* * * *} p<0.00001$, determined by one-way ANOVA. C)

927 Proposed model for suppression of the ALT pathway by the chromatin remodelling factor

928 ATRX.

929

930

931

932

933

934

935

936

937 
A

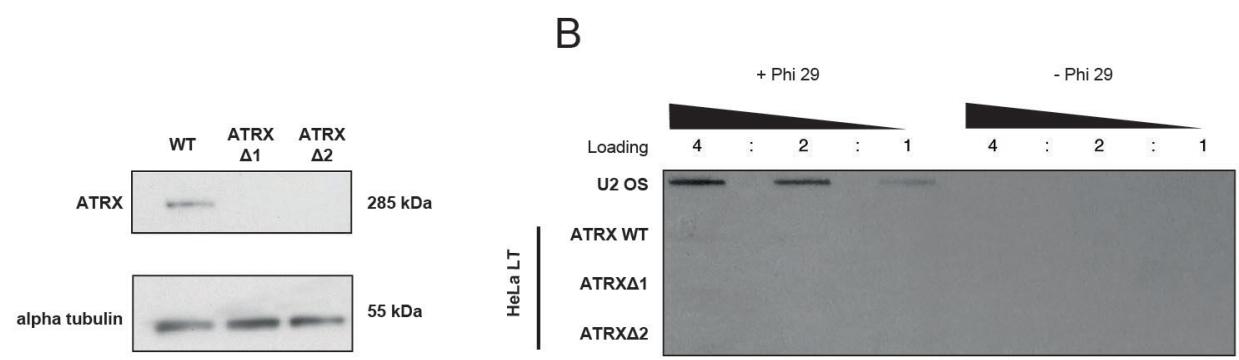

D

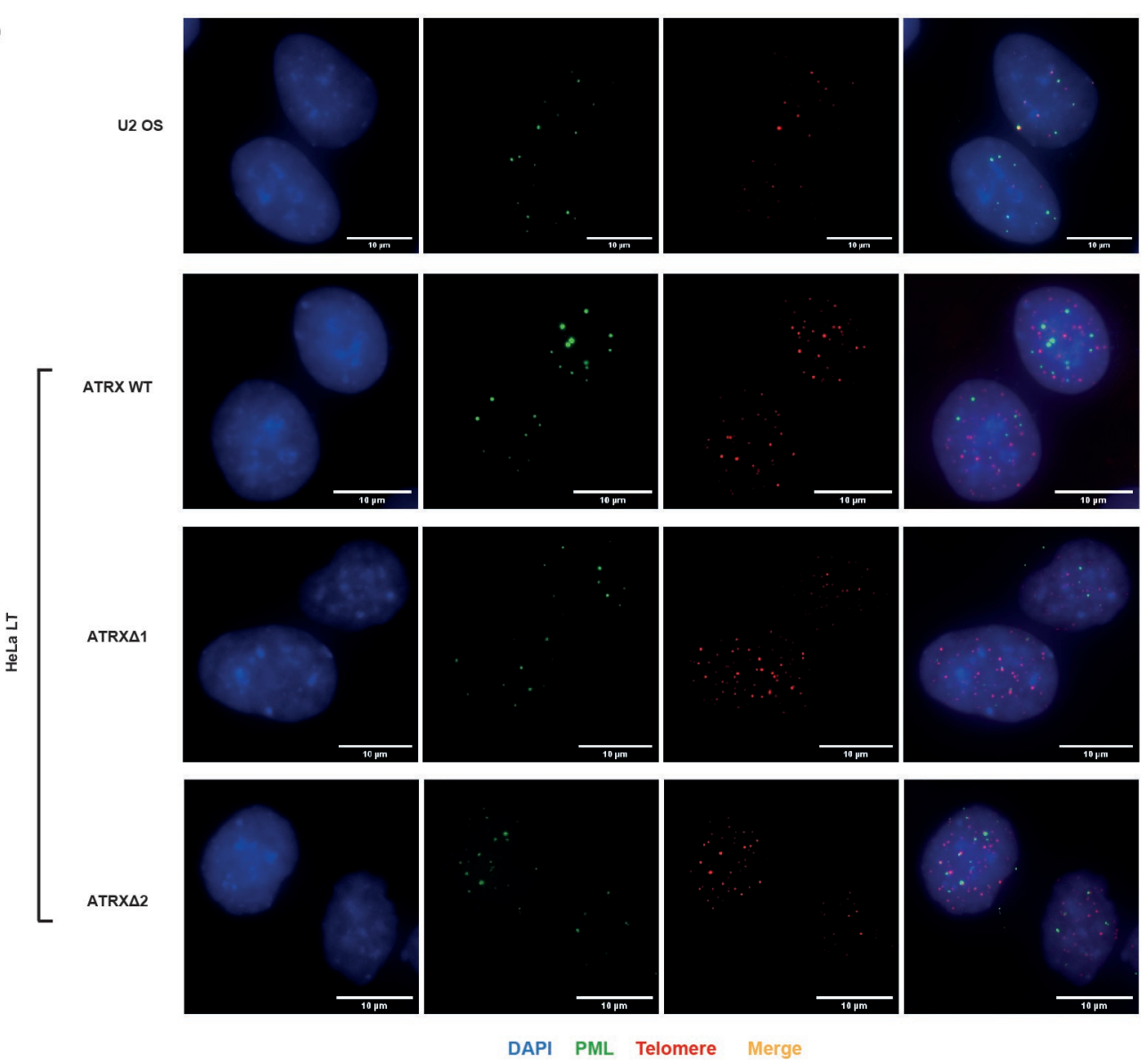

C

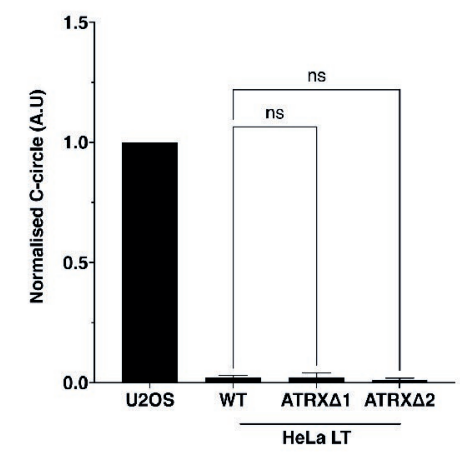

E

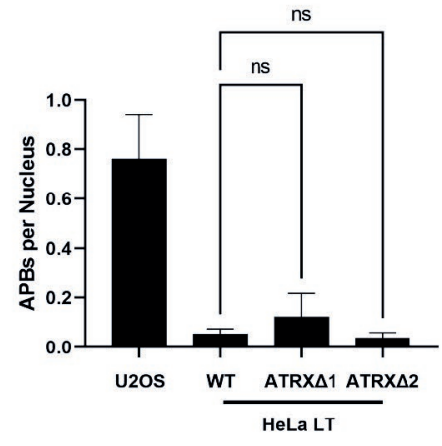

F

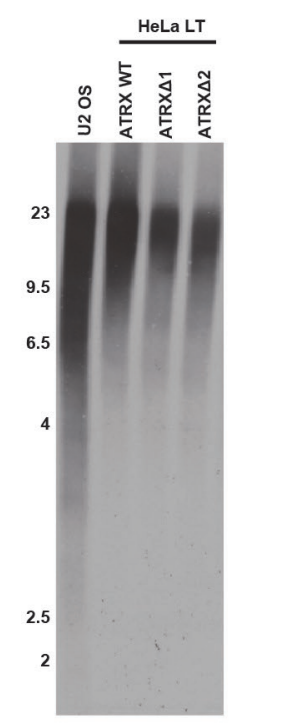


A

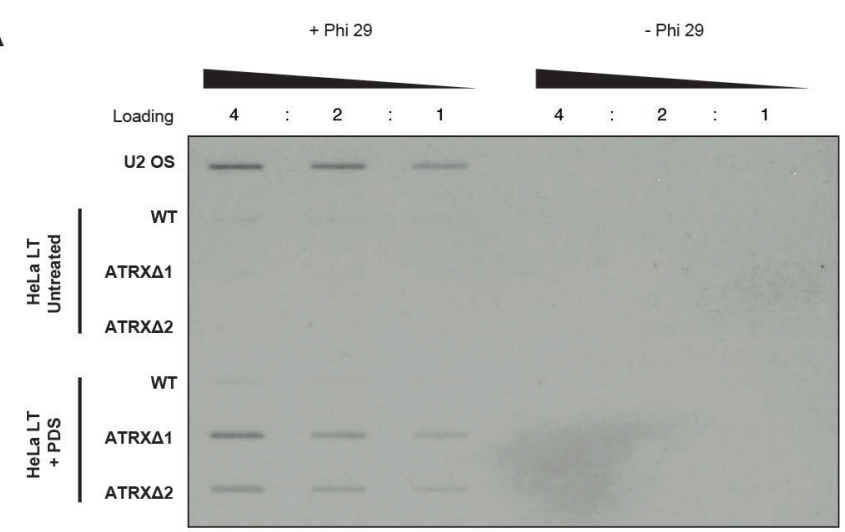

C
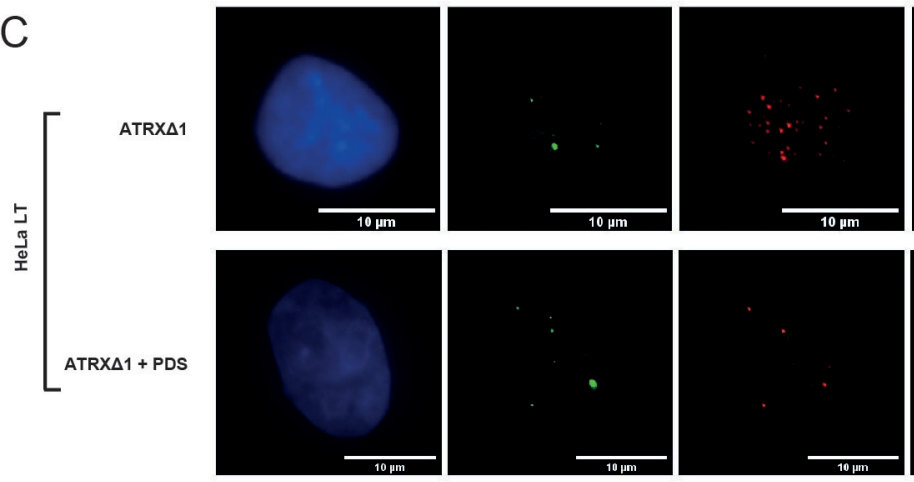

DAPI PML Telomere Merge

E
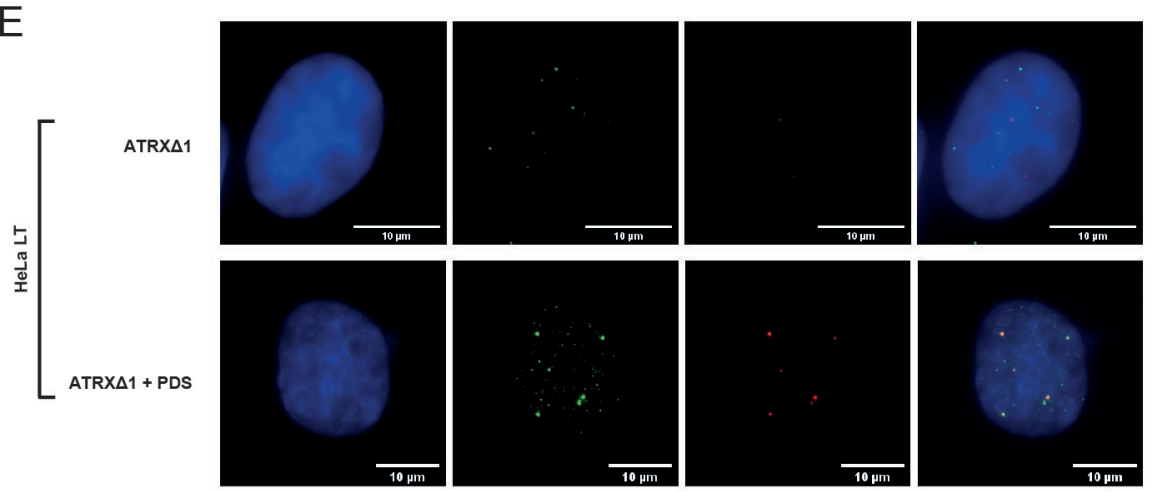

DAPI RPA Telomere Merge

G

G HeLa LT PDS
B

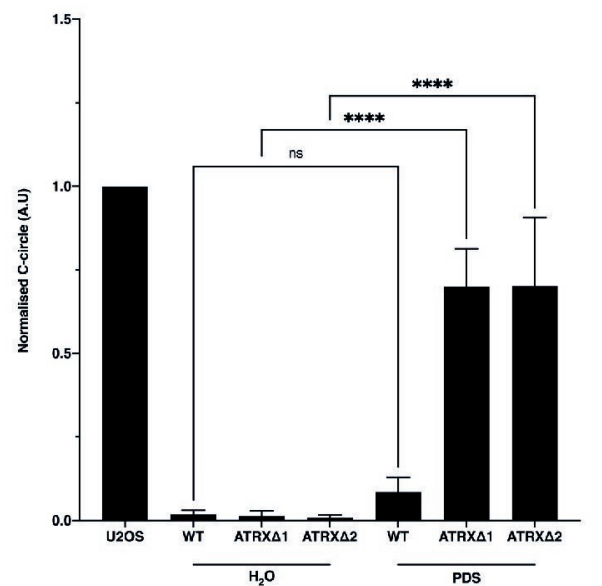

D

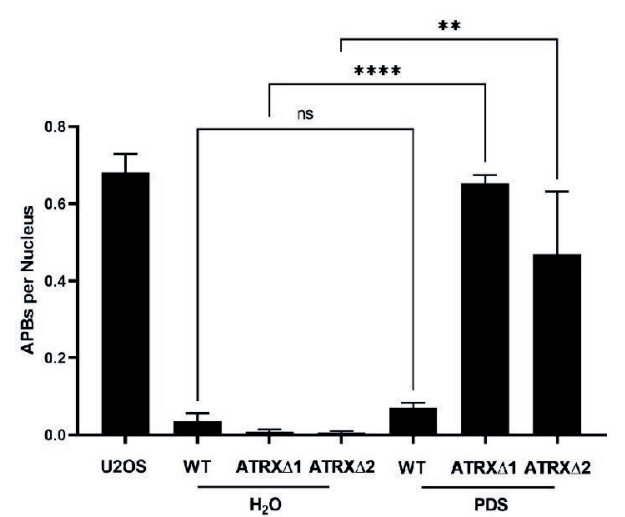

F

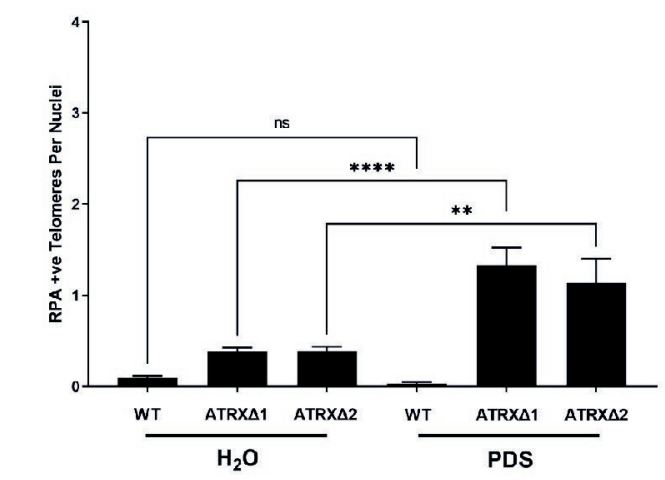

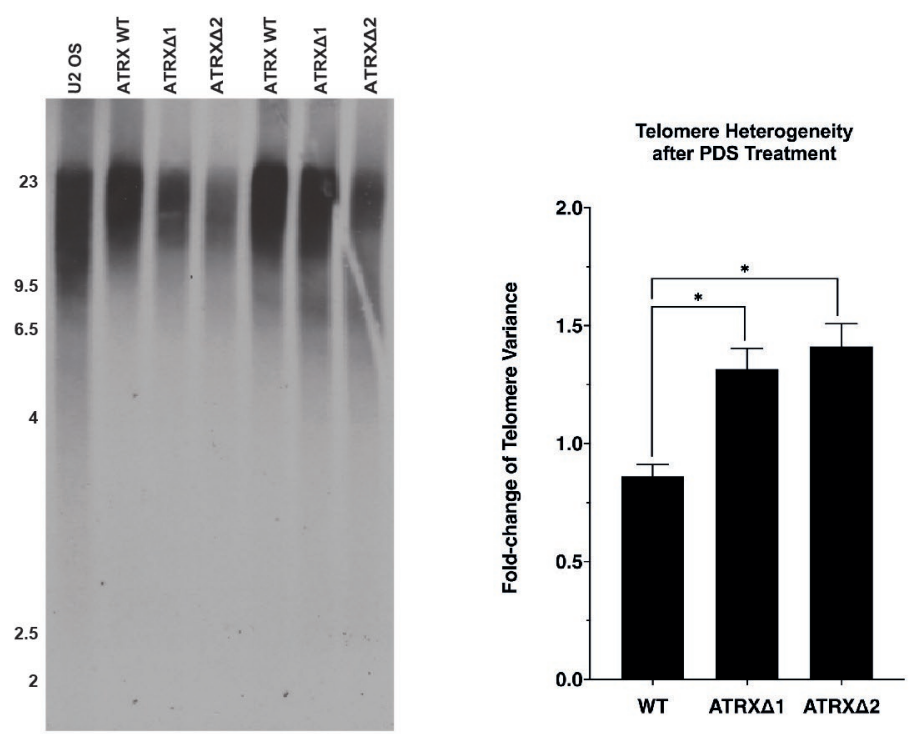


HeLa LT

A
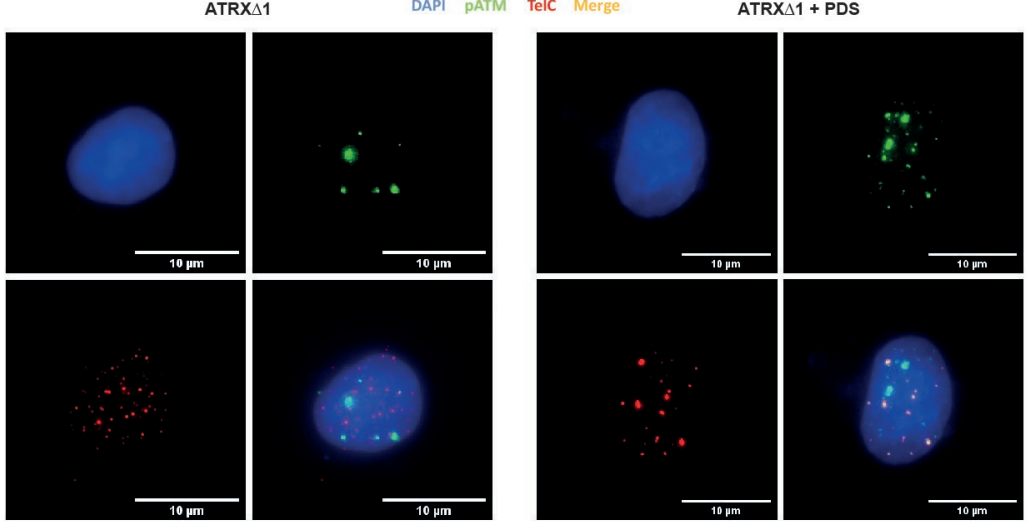

C

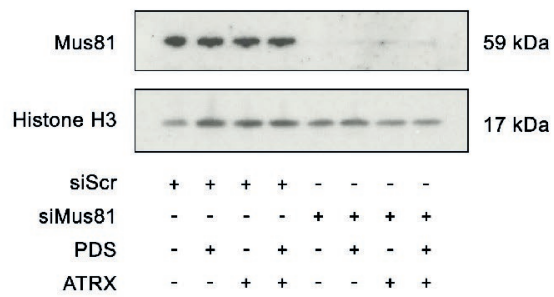

E

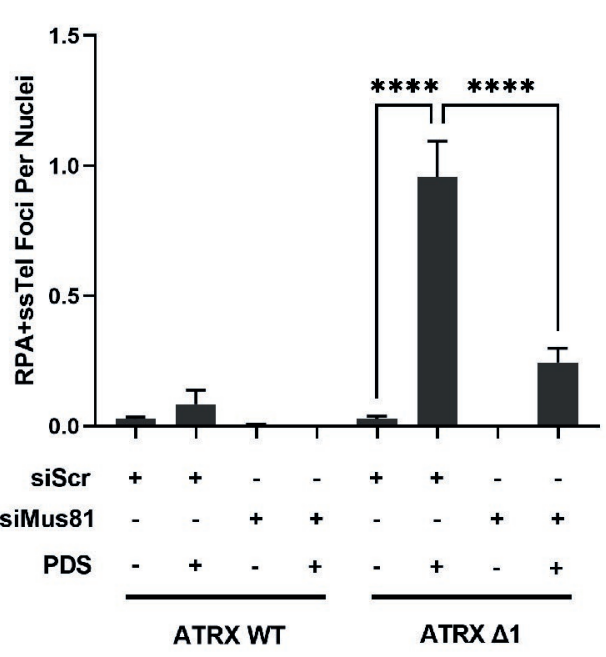

F

志
B
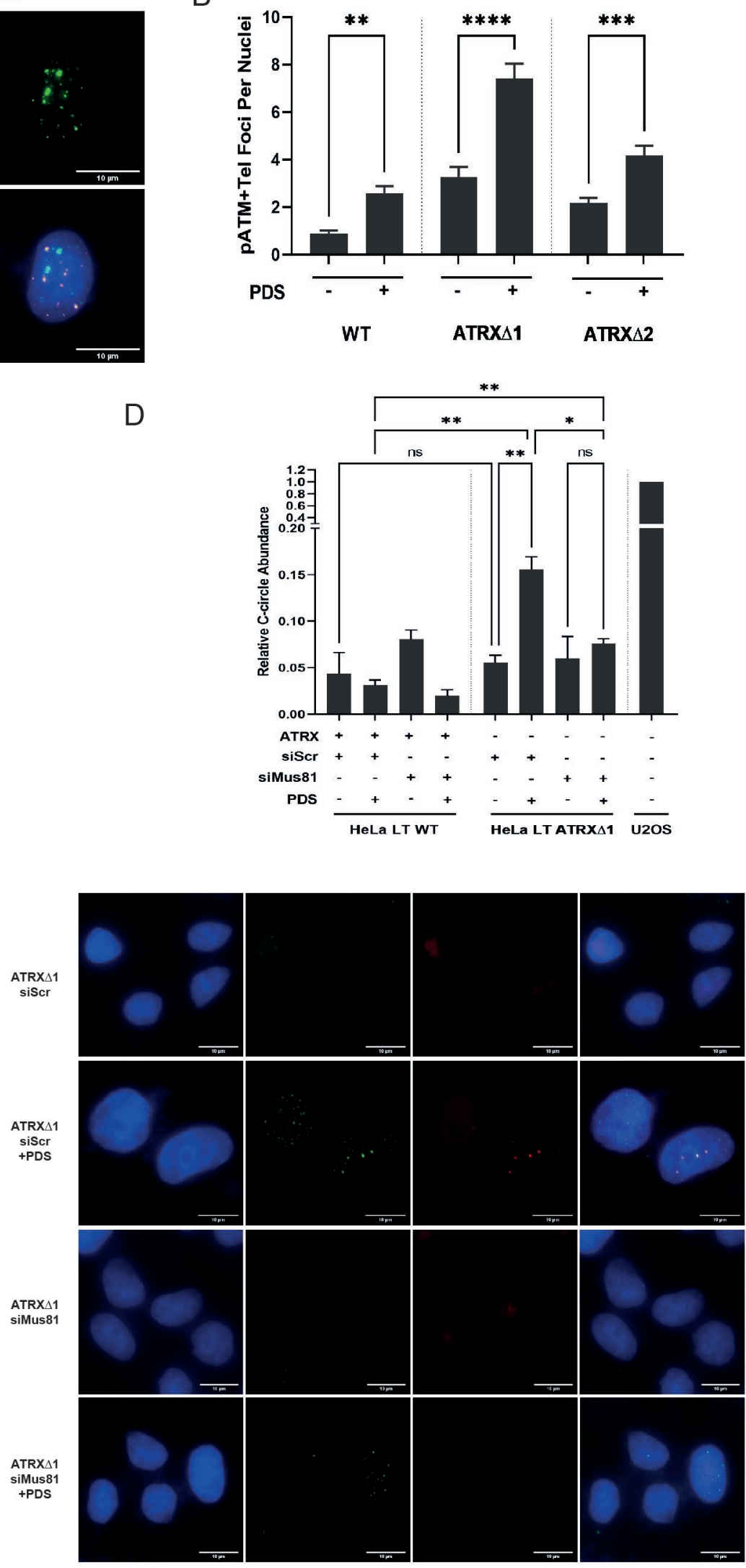

DAPI RPA Telc Merge 
A
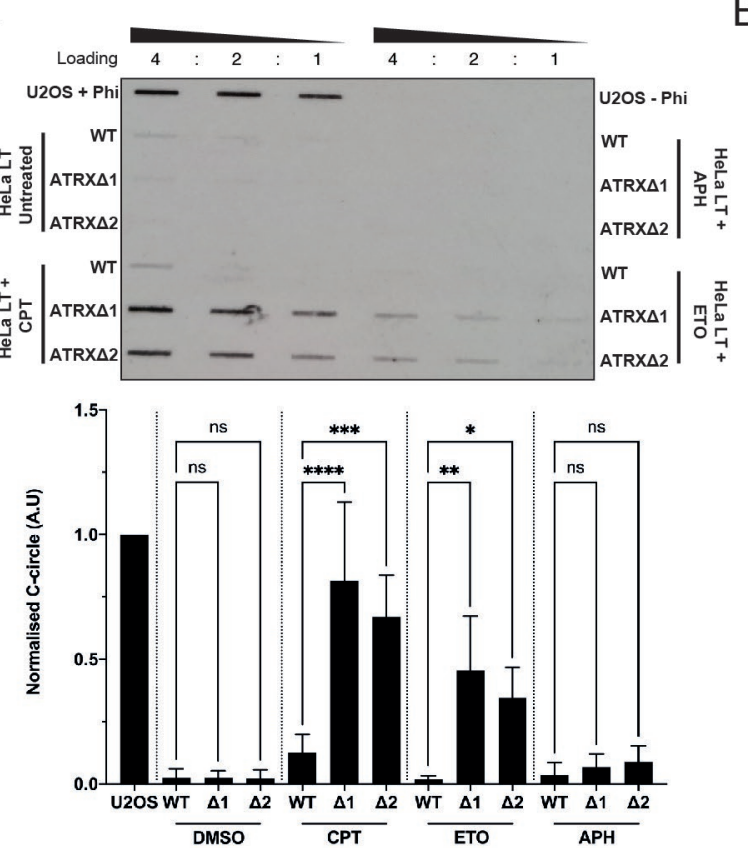

C
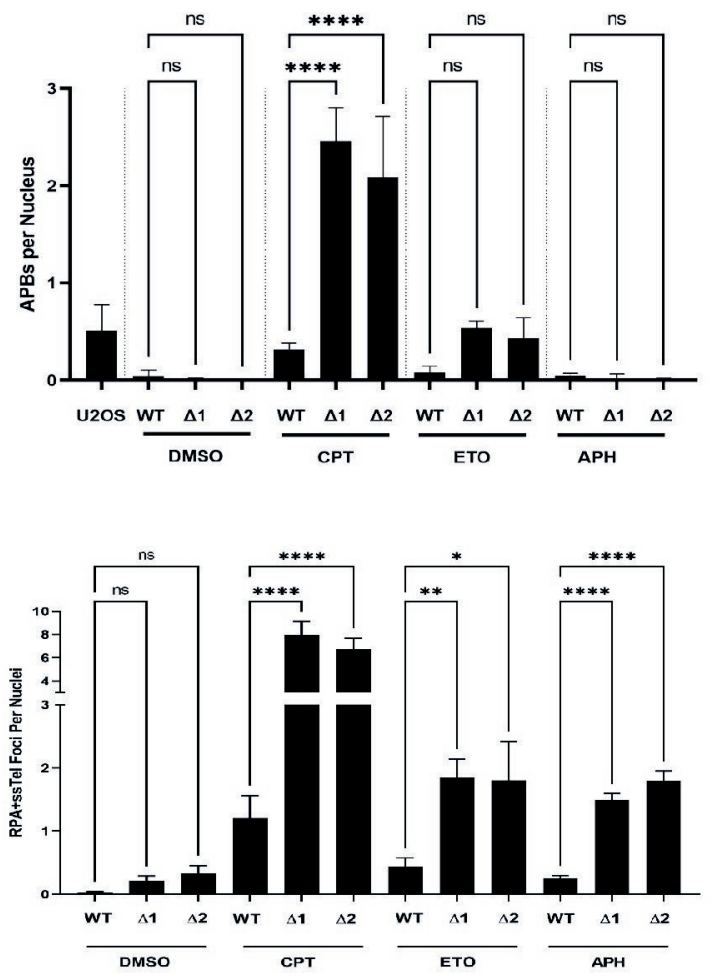

B

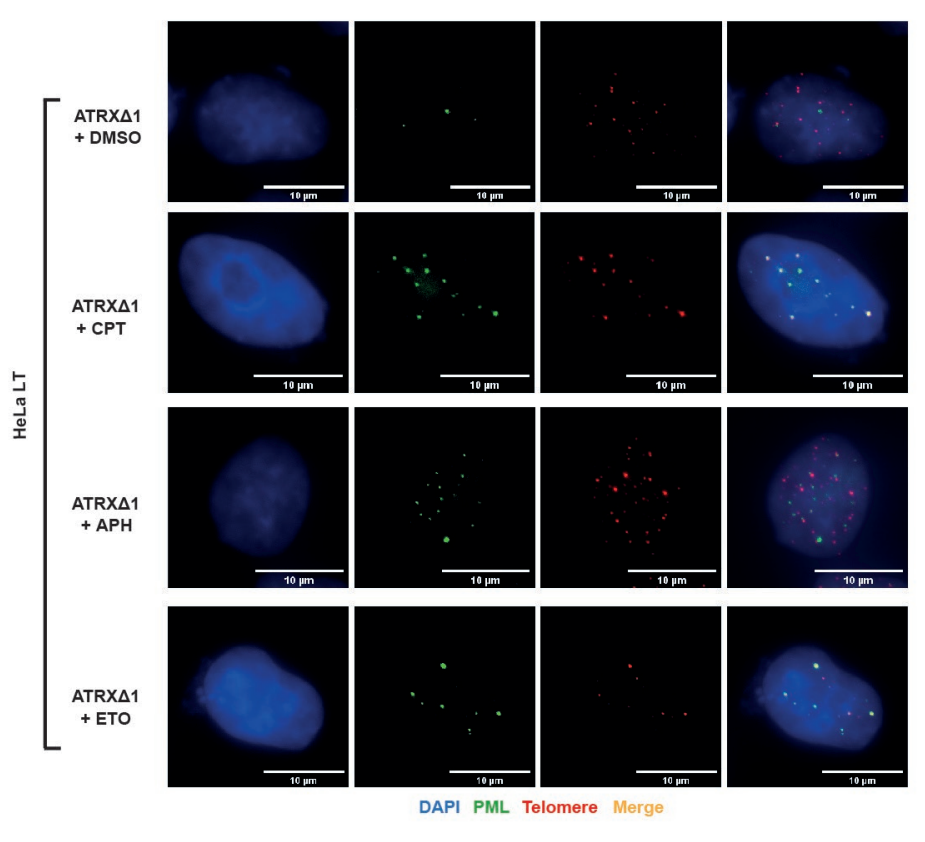

D

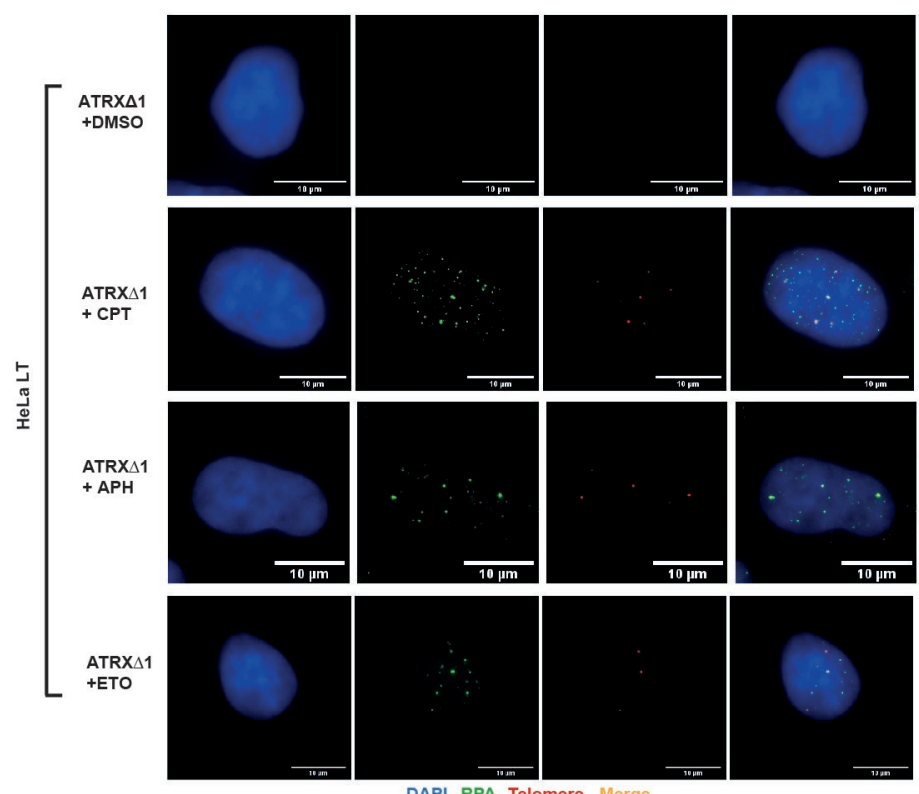

DAPI RPA Telomere Merge 
A

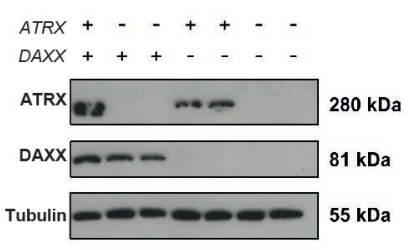

B
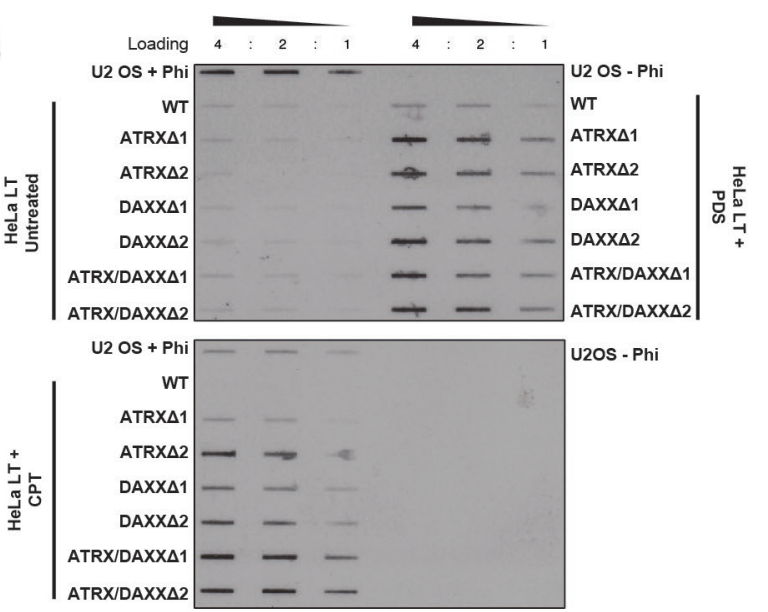

C
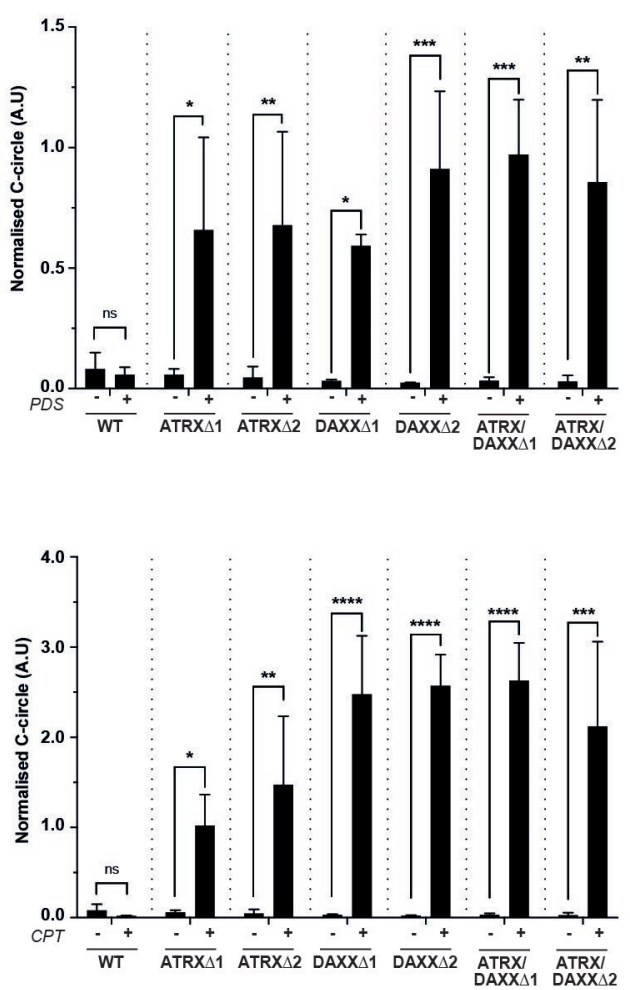

\section{$\mathrm{E}$}

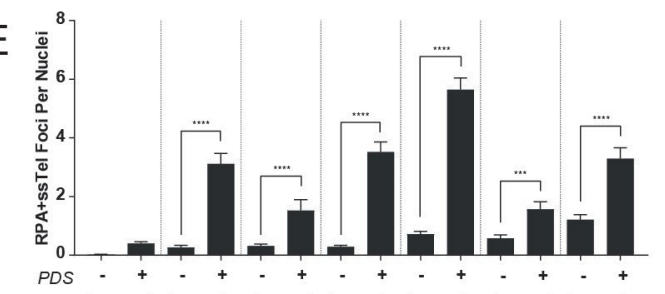

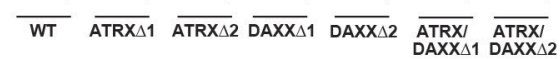

D

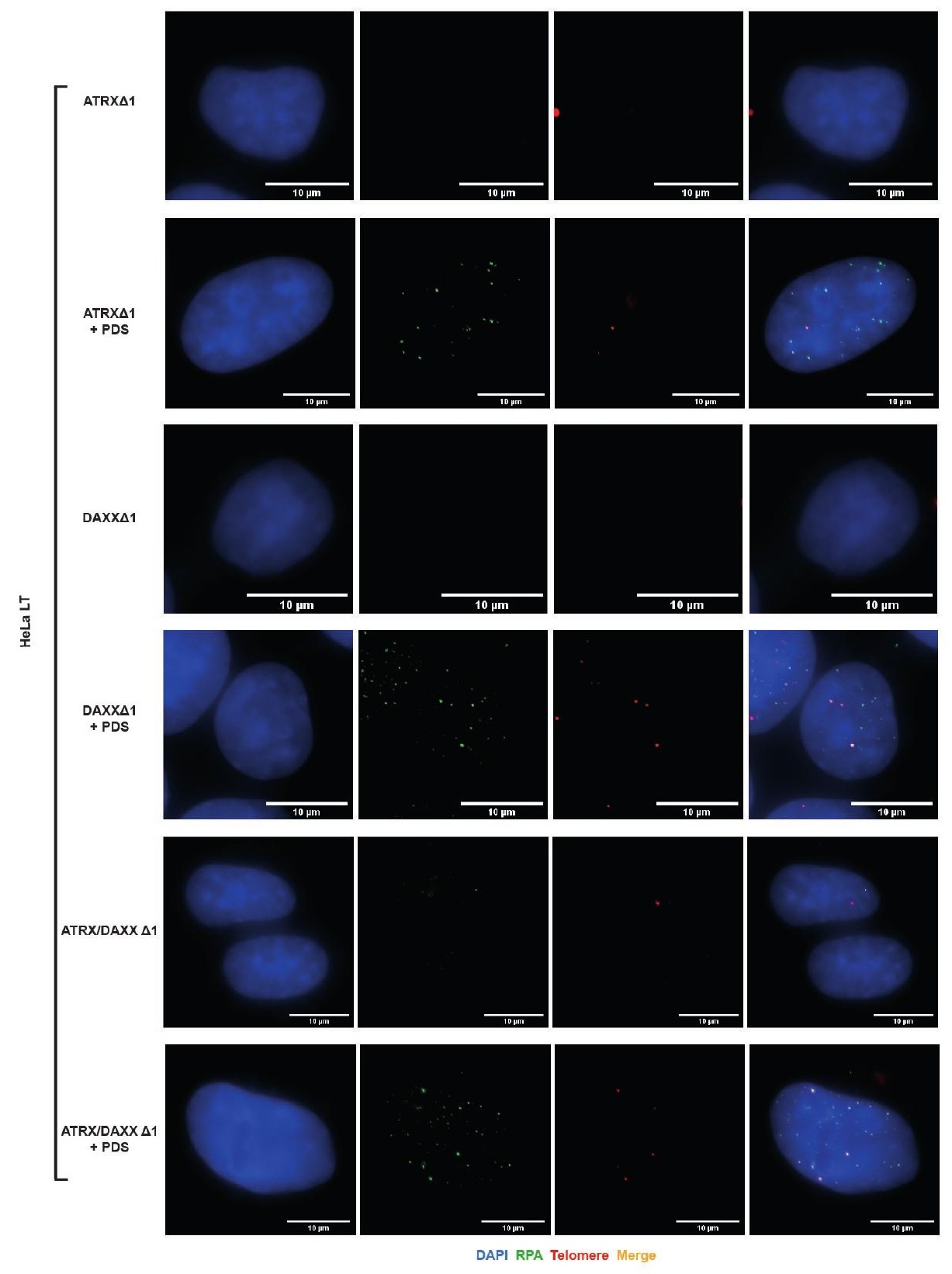

F

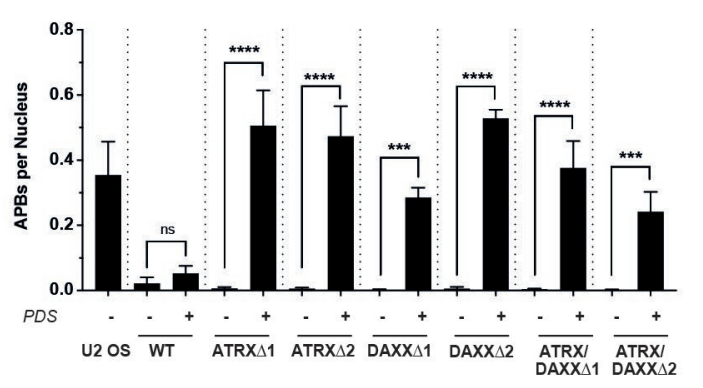

G

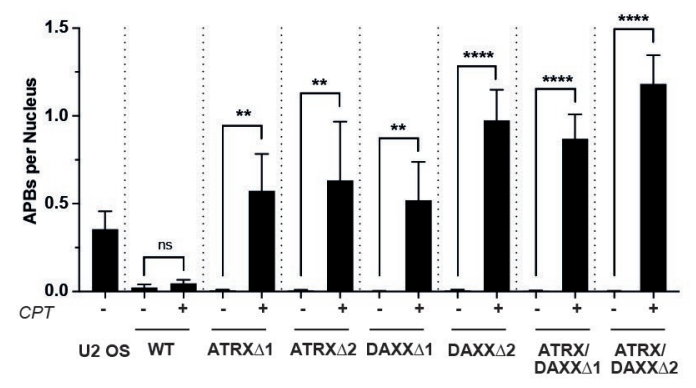


A

B

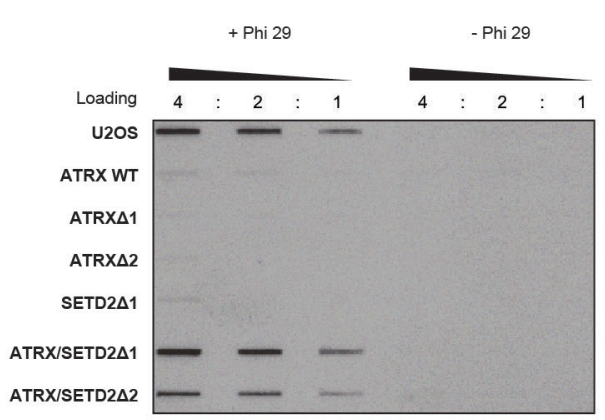

D

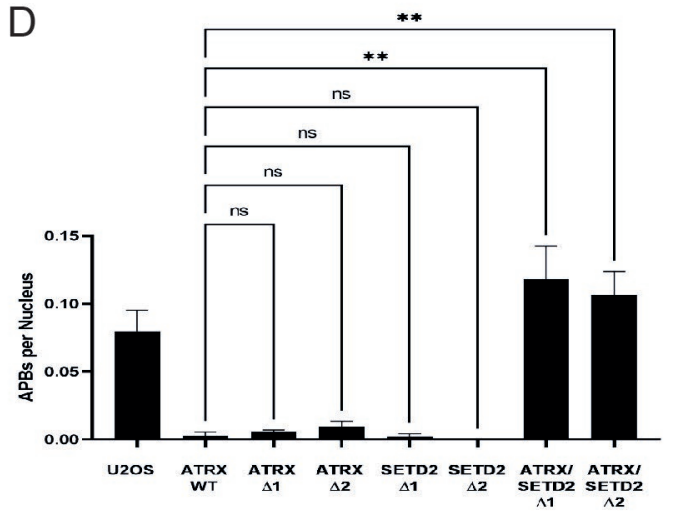

G

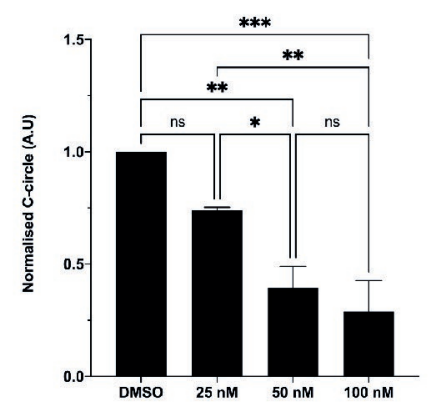

J

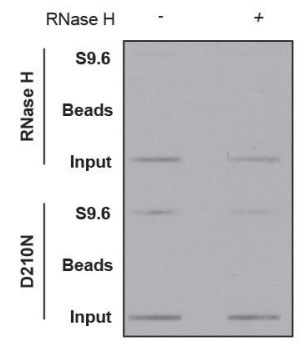

E

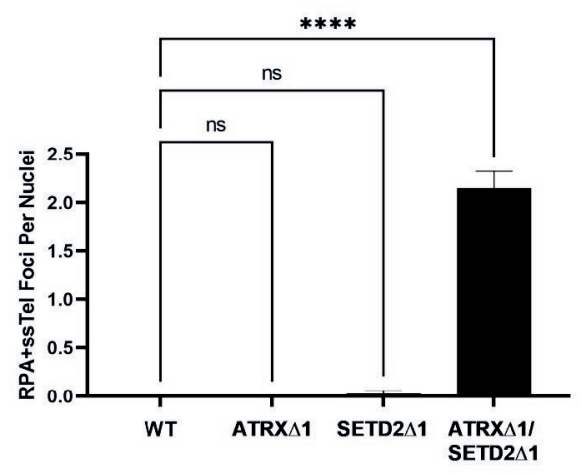

$\mathrm{H}$
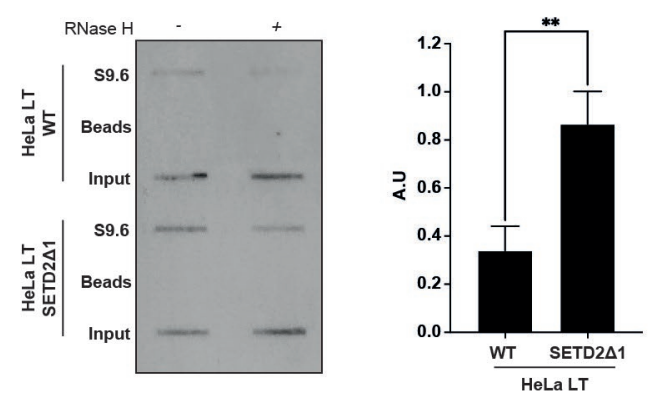

K

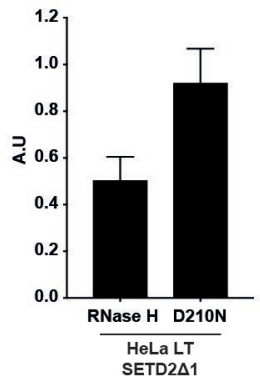

C

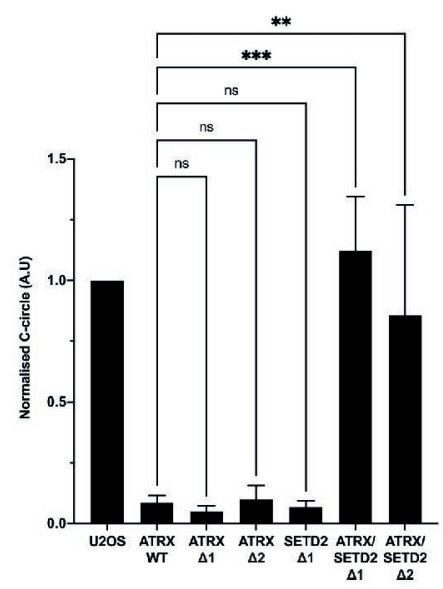

F

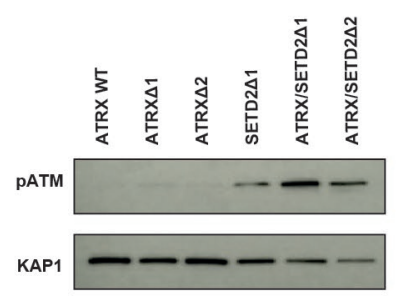

I
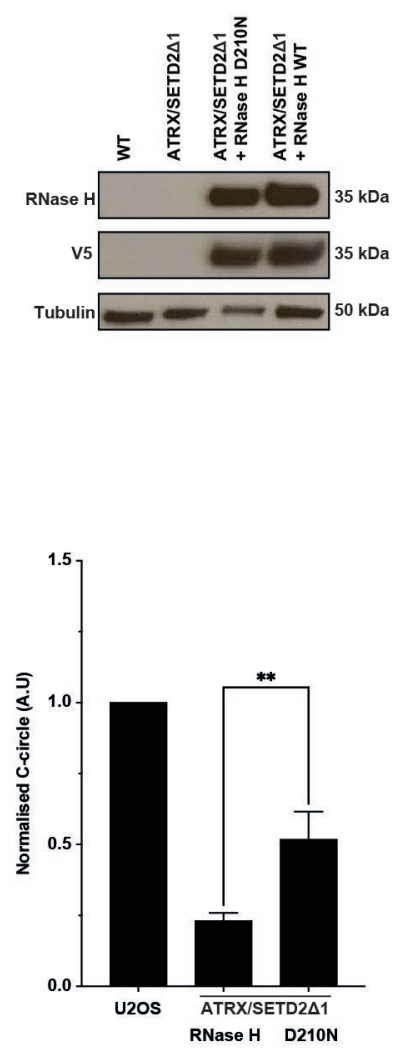
B
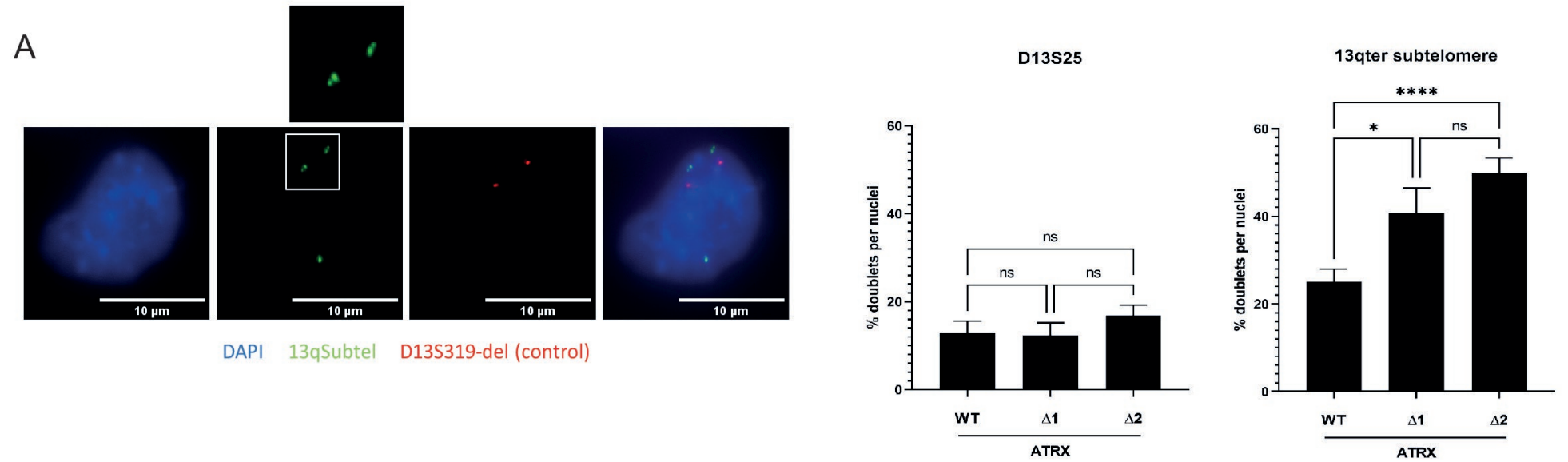

C

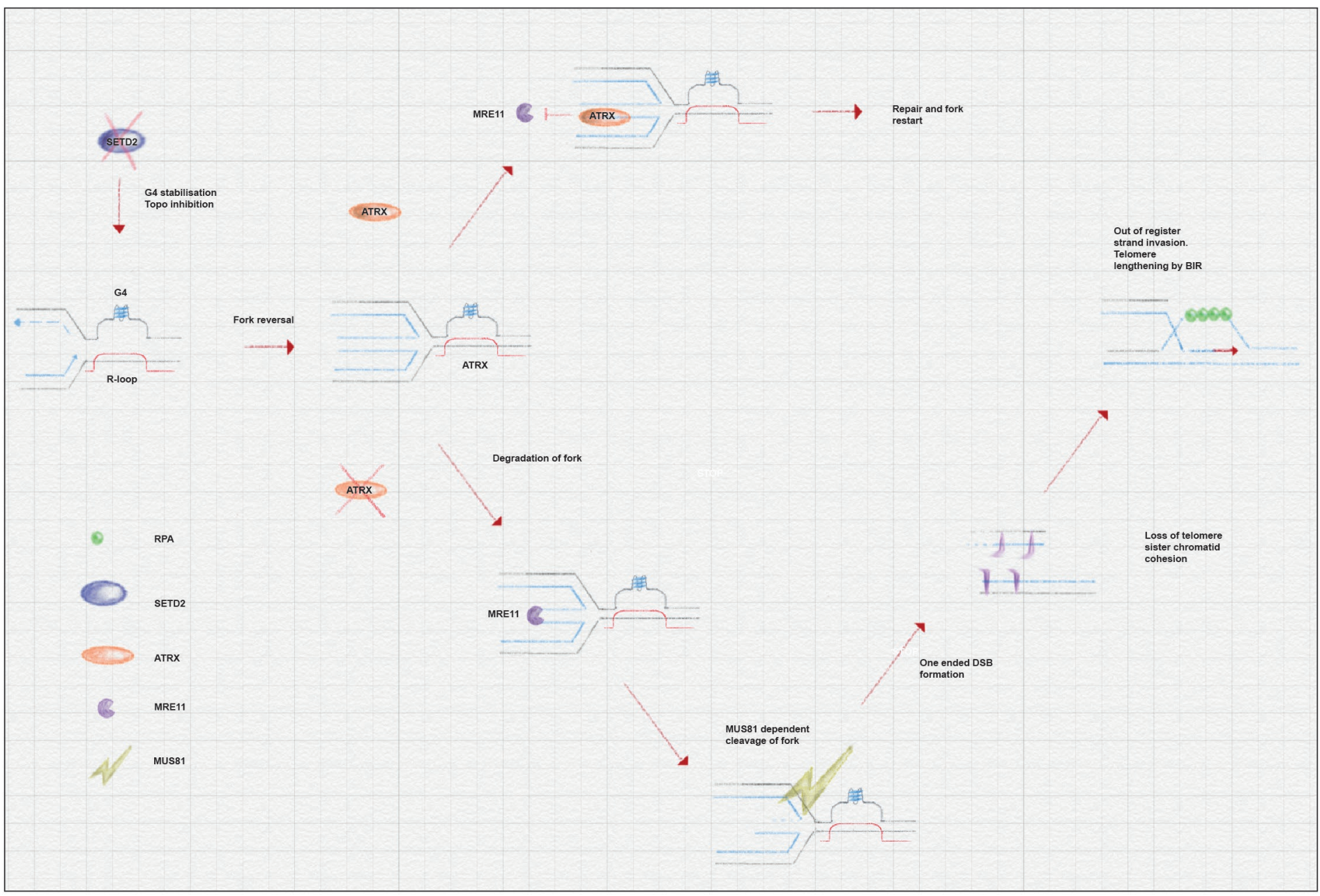




\section{SUPPLEMENTAL INFORMATION}

SUPPLEMENTARY TABLE 1 - List of Antibodies Used

\begin{tabular}{|c|c|c|}
\hline ANTIBODY & SOURCE & IDENTIFIER \\
\hline Alexa Fluor 488 Goat Anti-mouse & Invitrogen & $\begin{array}{l}\text { Cat\# A11029; } \\
2066710\end{array}$ \\
\hline Alexa Fluor 488 Goat Anti-rabbit & Invitrogen & $\begin{array}{l}\text { Cat\# A11034; } \\
2256692\end{array}$ \\
\hline Alexa Fluor 568 Goat Anti-mouse & Invitrogen & $\begin{array}{l}\text { Cat\# A11004; } \\
1906485\end{array}$ \\
\hline Alexa Fluor 568 Goat Anti-rabbit & Invitrogen & $\begin{array}{l}\text { Cat\# A11011; } \\
1871167\end{array}$ \\
\hline Anti-mouse IgG & Sigma & $\begin{array}{l}\text { Cat\# A9044; } \\
\text { 089M4797V }\end{array}$ \\
\hline Anti-rabbit IgG & Sigma & $\begin{array}{l}\text { Cat\# A6667; } \\
\text { SLBX8137 }\end{array}$ \\
\hline ATRX & Abcam & $\begin{array}{l}\text { Cat\# ab97508; } \\
\text { GR3313858-2 }\end{array}$ \\
\hline ATRX, clone 39F & Sigma & Cat\# MABE1798 \\
\hline DAXX & Sigma & $\begin{array}{l}\text { Cat\# D7810; } \\
\text { 084M4817V }\end{array}$ \\
\hline DIG & Roche & $\begin{array}{l}\text { Cat\# 32871922; } \\
11093274910\end{array}$ \\
\hline $\mathrm{H} 3$ & Biolegend & $\begin{array}{l}\text { Cat\# 819414; } \\
\text { B303830 }\end{array}$ \\
\hline H3k36me3 & Abcam & $\begin{array}{l}\text { Cat\# ab9050; } \\
\text { GR3307136-2 }\end{array}$ \\
\hline KAP1 & Abcam & Cat\# ab10483 \\
\hline MUS81 & Santa Cruz & $\begin{array}{l}\text { Cat\# sc-53382; } \\
\text { Clone MTA30 2G- } \\
10 / 3\end{array}$ \\
\hline pATM-S1981 & Santa Cruz & $\begin{array}{l}\text { Cat\# sc-47739; } \\
\text { 10H11.E12 }\end{array}$ \\
\hline PML & Santa Cruz & Cat\# dc-966; I1218 \\
\hline PML & Santa Cruz & $\begin{array}{l}\text { Cat\# sc-5621; } \\
\text { D2314 }\end{array}$ \\
\hline PML & Abcam & $\begin{array}{l}\text { Cat\# ab53773; } \\
\text { GR317758-18 }\end{array}$ \\
\hline RNase $\mathrm{H}$ & Santa Cruz & $\begin{array}{l}\text { Cat\# sc-101114; } \\
\text { D2313 }\end{array}$ \\
\hline RPA-2 & Abcam & $\begin{array}{l}\text { Cat\# ab2175; } \\
\text { GR3224197-4 }\end{array}$ \\
\hline S9.6 & Sigma & Cat\# MABE1095 \\
\hline
\end{tabular}




\begin{tabular}{|l|l|l|}
\hline SETD2 & GeneTex & Cat\# GTX127905 \\
\hline Tubulin & Abcam & $\begin{array}{l}\text { Cat\# ab7291; } \\
\text { GR3197113-3 }\end{array}$ \\
\hline V5 & Cell Signal & $\begin{array}{l}\text { Cat\# 13202S; } \\
\text { D3H8Q }\end{array}$ \\
\hline
\end{tabular}

5

6 
8

\begin{tabular}{|l|l|l|}
\hline CELL LINE & SOURCE & IDENTIFIER \\
\hline HeLa LT & O'Sullivan et al. 2014 & N/A \\
\hline HeLa LT ATRX 1 & This Paper & N/A \\
\hline HeLa LT ATRX 2 & This Paper & N/A \\
\hline HeLa LT DAXX 1 N & This Paper & N/A \\
\hline HeLa LT DAXX 2 & This Paper & N/A \\
\hline HeLa LT ATRX/DAXX 1 & This Paper & N/A \\
\hline HeLa LT ATRX/DAXX 2 & This Paper & N/A \\
\hline HeLa LT SETD2 1 & This Paper & N/A \\
\hline HeLa LT ATRX/SETD2 1 & This Paper & N/A \\
\hline HeLa LT ATRX/SETD2 2 & This Paper & N/A \\
\hline HeLa ST (HeLa H3.3 SNAP) & Adam et al. 2013 & N/A \\
\hline G292 & ATCC & N/A \\
\hline SAOS-2 & ATCC & N/A \\
\hline U-2 OS & ATCC & N/A \\
\hline WI38 VA13 2RA & ATCC & N/A \\
\hline
\end{tabular}


11

\begin{tabular}{|l|l|l|}
\hline OLIGONUCLEOTIDE & SOURCE & IDENTIFIER \\
\hline $\begin{array}{l}\text { hTeloG: } \\
\text { ACACTAAGGTTTGGGTTTGGGTTTGGGTTTGGGTTAG } \\
\text { TGT }\end{array}$ & Life Tech & N/A \\
\hline $\begin{array}{l}\text { hTeloC: } \\
\text { TGTTAGGTATCCCTATCCCTATCCCTATCCCTATCCCTA } \\
\text { ACA }\end{array}$ & Life Tech & N/A \\
\hline $\begin{array}{l}\text { GlobinF: } \\
\text { CGGCGGCGGGCGGCGCGGGCTGGGCGGCTTCATCCA } \\
\text { CGTTCACCTTG }\end{array}$ & Life Tech & N/A \\
\hline $\begin{array}{l}\text { GlobinR: } \\
\text { GCCCGGCCCGCCGCGCCCGTCCCGCCGGAGGAGAAG } \\
\text { TCTGCCGTT }\end{array}$ & Life Tech & N/A \\
\hline $\begin{array}{l}\text { ON-TARGETplus Mus81 siRNA SmartPool } \\
\text { TelC DIG Probe: 5'DIG-TTAGGGTTAGGGTTAGGG }\end{array}$ & Dharmacon & L-016143-01-0005 \\
\hline D13S25, 13q14.3, Red & CytoCell & N/A \\
\hline 13qter, 13q34, Green & CytoCell & LPH 043 \\
\hline
\end{tabular}


Supplementary Figure 1. HeLa LT ATRX KO cells show sensitivity to PDS, APH and HU.

CellTiterGlo assay following treatment of HeLa LT ATRX Wild Type and KO cells with A) PDS,

B) $\mathrm{APH}$ and $\mathrm{C}) \mathrm{HU}$ at the doses shown. IC50 values are indicated, $\mathrm{n}=3$.

Supplementary Figure 2. PDS Treatment Induces ATRX Foci Formation. A) ImmunoFISH assay quantification showing treatment of HeLa LT WT cells with PDS induces an increase in ATRX foci formation at telomeres, $>100$ nuclei analysed across 2 biological replicates. ${ }^{* * * *} p<$ 0.00001 , determined by unpaired Student's t test. B) Representative ImmunoFISH images.

Supplementary Figure 3. Treatment with PDS in ATRX KO cells leads to telomere clustering.

A) Intensity of telomere foci. Foci considered intense are shown above dotted line 15000 (a.u.). Significance donates increase in intense foci. Data is collation of 3 biological replicates each with over 100 nuclei analysed. $* * * * p<0.00001$, determined by one-way ANOVA. B) Quantitation of telomere number per nucleus. Data is collation of 3 biological replicates each with over 150 nuclei analysed. $* * * p<0.00001$.

Supplementary Figure 4. RPA coincident with single stranded telomeric sequence is a marker of ALT activity. A) Representative image showing RPA coincident with telomeric sequence in ALT positive U-2 OS and WI38 VA13 2RA cells, >100 nuclei analysed across 3 biological replicates. B) Quantification of RPA+ssTel in a panel of ALT, non-ALT, and ATRX reexpressed cell lines. ${ }^{* * * *} \mathrm{p}<0.00001$, determined by one-way ANOVA. C) WI38 VA13 2RA cells treated with RNaseA and $\mathrm{RNaseH}$ did not show reduction in telomere foci, $>50$ nuclei analysed across 2 biological replicates. ns signifies $p>0.05$, determined by one-way ANOVA.

Supplementary Figure 5. Telomere length and heterogeneity increases following treatment with PDS amd CPT. A) Ratio of telomere repeats to the single copy gene beta-globin (T/S ratio) following the addition of PDS as assessed by mm-qPCR in HeLa LT ATRX wildtype or ATRX KO clones, $\mathrm{n}=6 .{ }^{*} \mathrm{p}<0.05,{ }^{* * *} \mathrm{p}<0.0001$, determined by unpaired Student's $\mathrm{t}$ test. B) Terminal restriction fragment (TRF) assay following treatment with CPT. Telomere length 
heterogeneity was calculated using TeloMetric software, $n=2 .{ }^{* *} p<0.001$, determined by 46 one-way ANOVA.

47

48 Supplementary Figure 6. Treatment with PDS in combination with ATRX loss triggers ALT markers in HeLa standard telomere length (ST) cells. A) Immunoblot of shScr and shATRX populations in HeLa ST cells. B-C) C-circle blot and quantification showing HeLa ST shScr and shATRX with CPT treatment at indicated doses in $n M, n=2$. D-E) RPA ssTel representative images and quantification showing HeLa H3.3 SNAP shScr and shATRX with PDS treatment,

$53>100$ nuclei analysed across 3 biological replicates. $* p<0.05, * * * * p<0.00001$, determined by one-way ANOVA.

Supplementary Figure 7. Effect of Triptolide Treatment on Cell Cycle. A) Representative Ccircle assay in HeLa LT ATRX/SETD2 knockout cells after 24 hours treatment with 25, 50 and 100 nM Triptolide. B-E) Representative plots of PI staining for the doses of triptolide indicated. F) Quantification of cell cycle phases by PI staining and FACs analysis upon treatment of indicated doses of triptolide in the HeLa LT ATRX/SETD2 knockout cells, $n=2$. 
A

$\rightarrow \quad$ HeLa-LT WT: $\frac{\mathrm{IC}_{50}}{244.1 \mathrm{nM}}$

- Hela-LT ATRX KO-c1: $155.8 \mathrm{nM}$

* HeLa-LT ATRX KO-c2: $149.5 \mathrm{nM}$

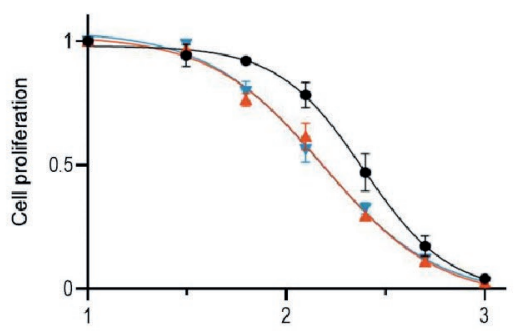

$\log _{10}[$ Pyridostatin (nM)]

C

- $\quad$ HeLa-LT WT: $\frac{10_{50}}{251.3 \mu \mathrm{M}}$

^ HeLa-LT ATRX KO-c1: $194.9 \mu \mathrm{M}$

$\rightarrow$ HeLa-LT ATRX KO-C2: $194.3 \mu \mathrm{M}$

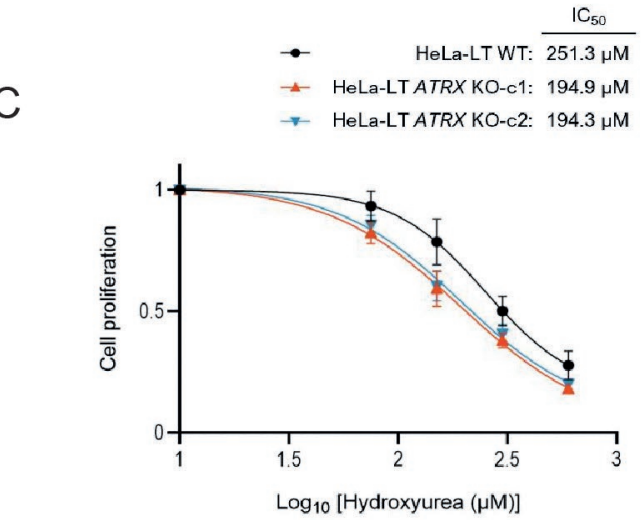

B

-- $\quad$ HeLa-LT WT: $\frac{\mathrm{IC}_{50}}{722.9 \mathrm{nM}}$

- HeLa-LT ATRX KO-c1: $503.2 \mathrm{nM}$

$\rightarrow$ HeLa-LT ATRX KO-c2: $375.1 \mathrm{nM}$

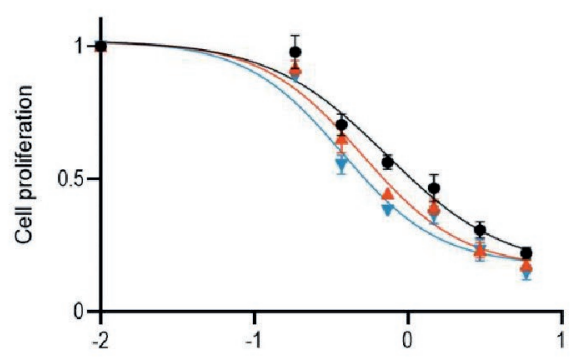

$\log _{10}[$ Aphidicolin $(\mu \mathrm{M})]$ 
FIGURE S2

A

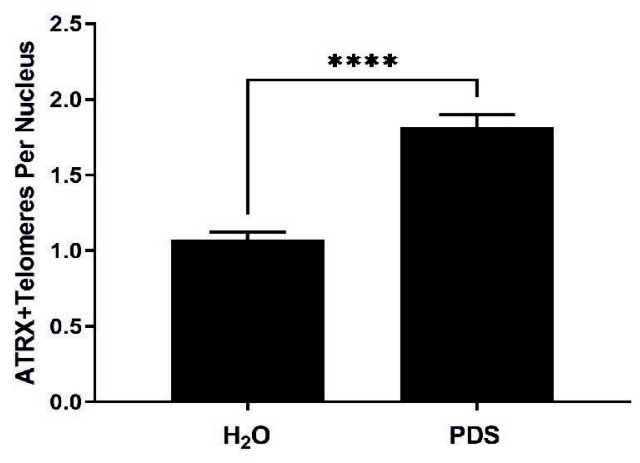

B

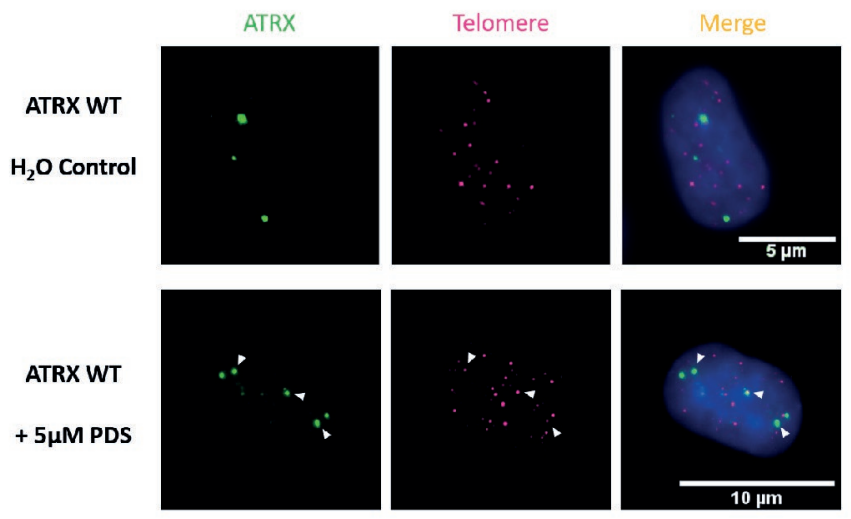


A

Telomere Intensity

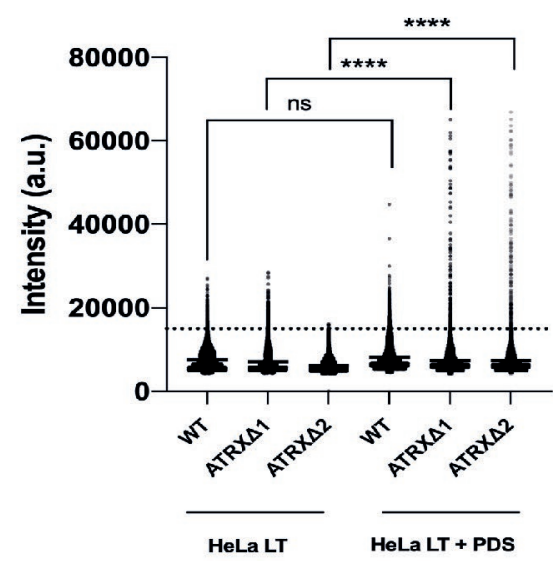

B

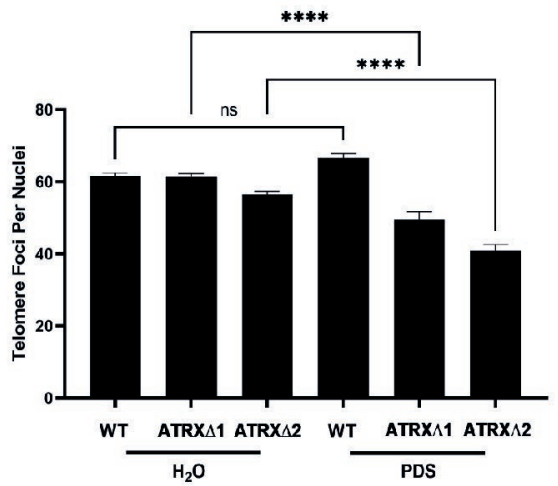




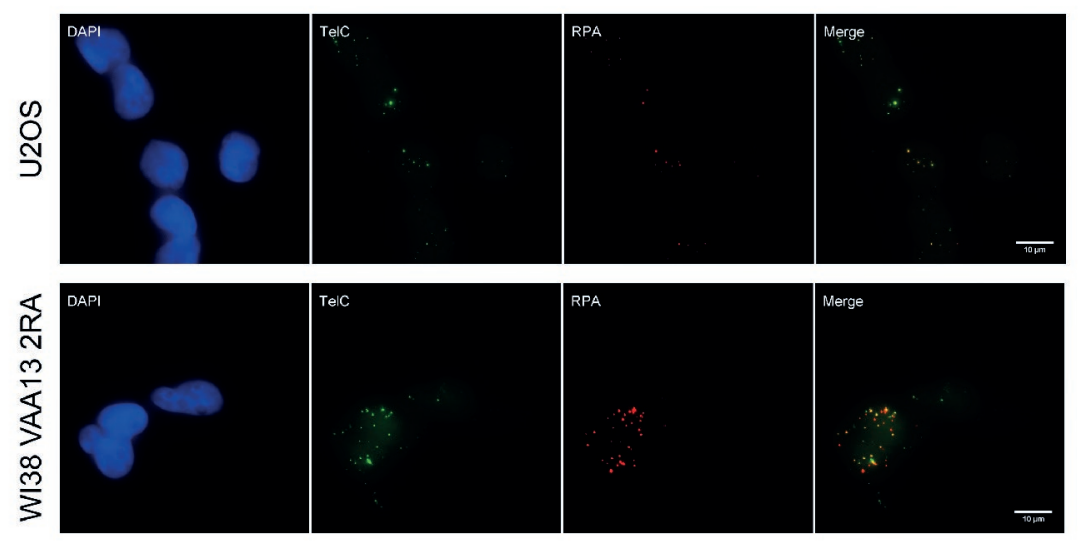

B

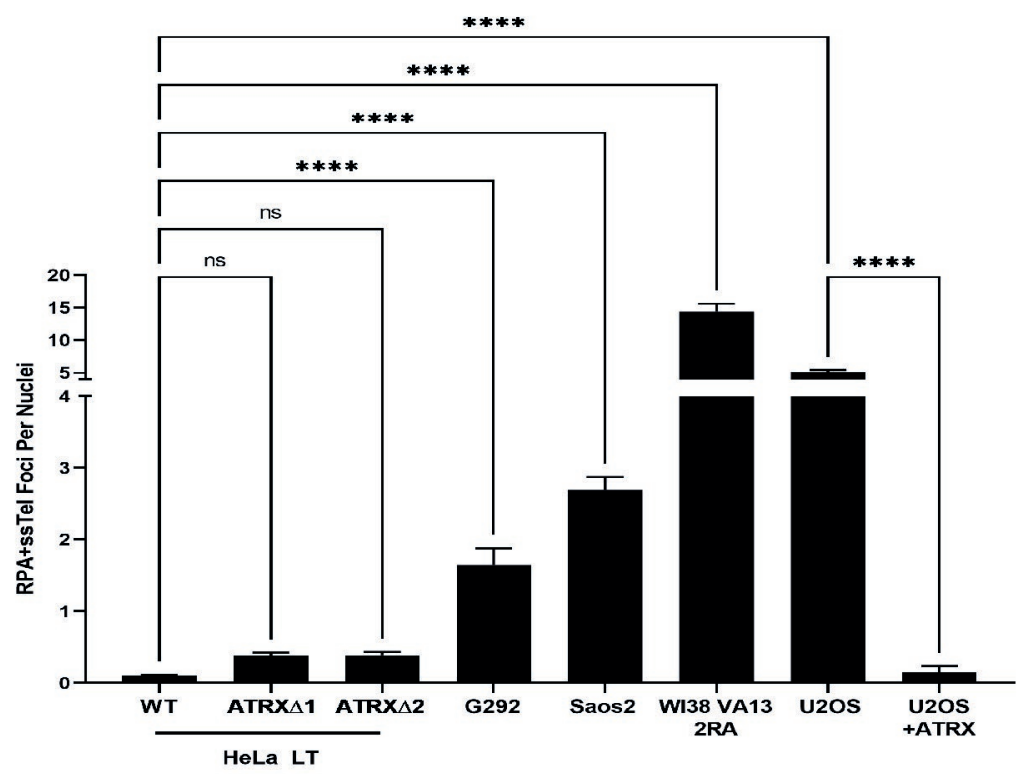

C
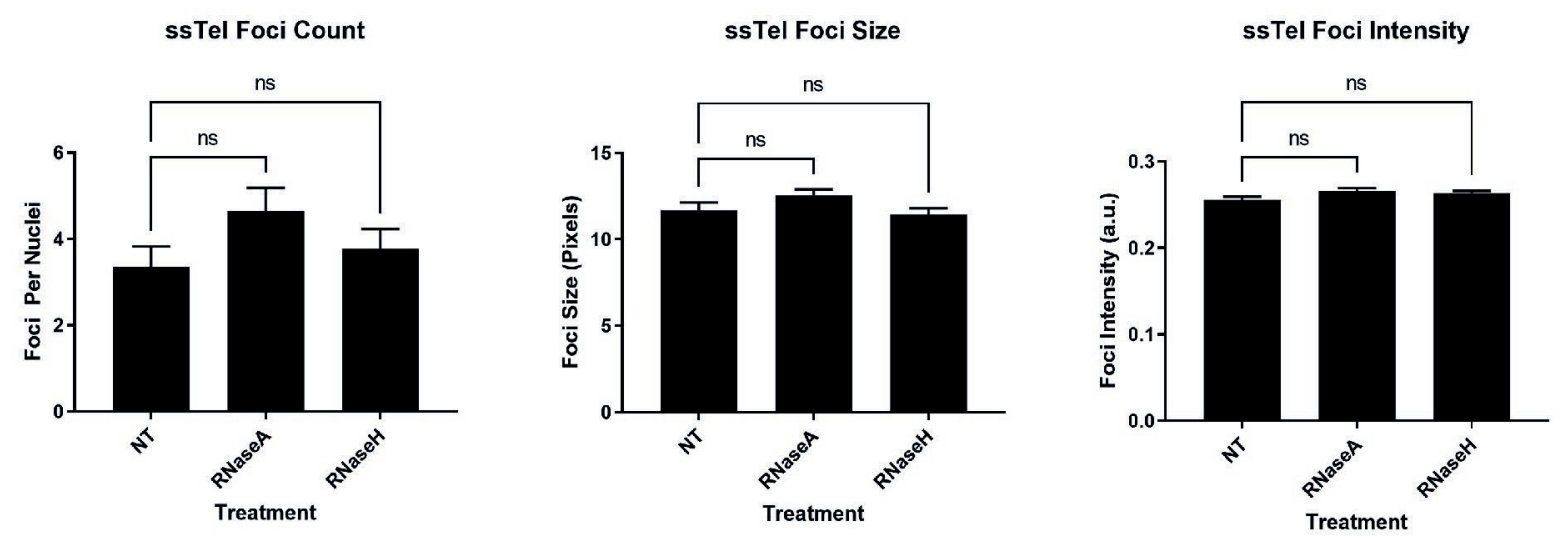
A

T/S Ratios [Globin]

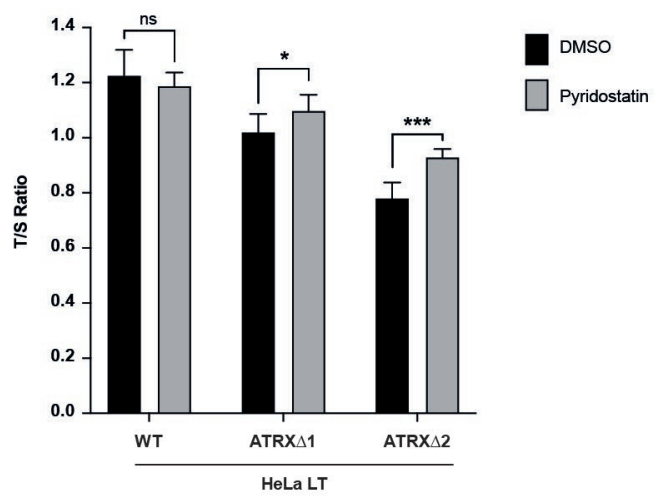

B
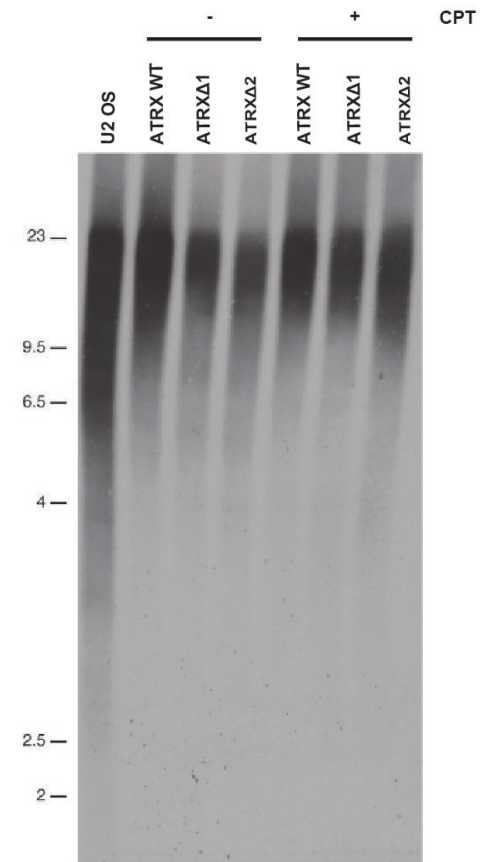

Telomere Heterogeneity after CPT Treatment

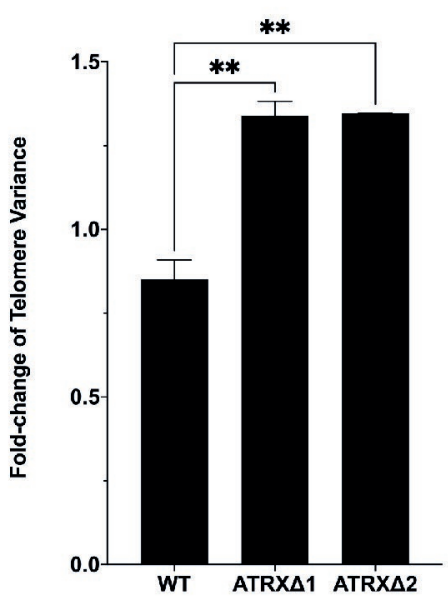


FIGURE S6

A

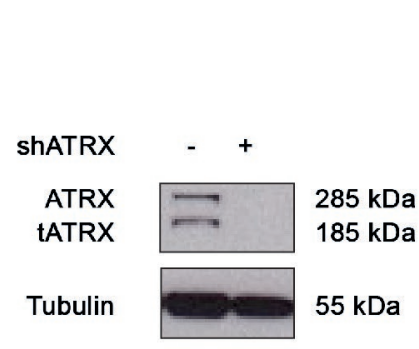

D

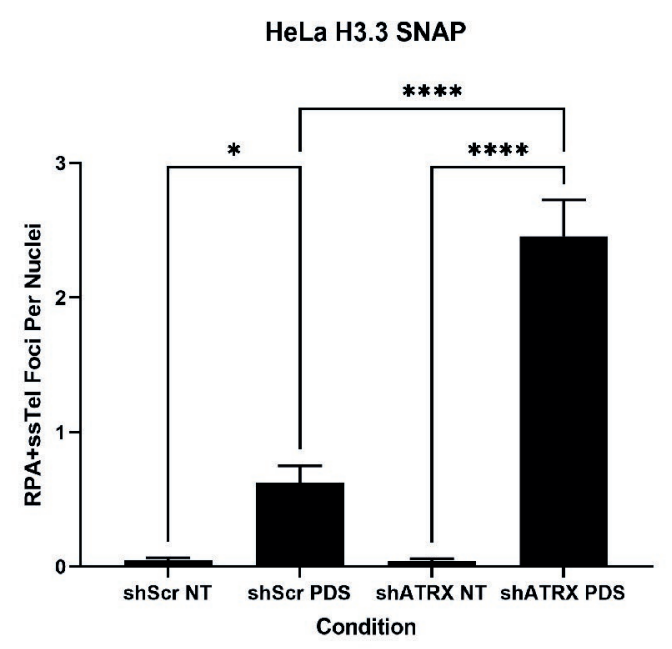

B

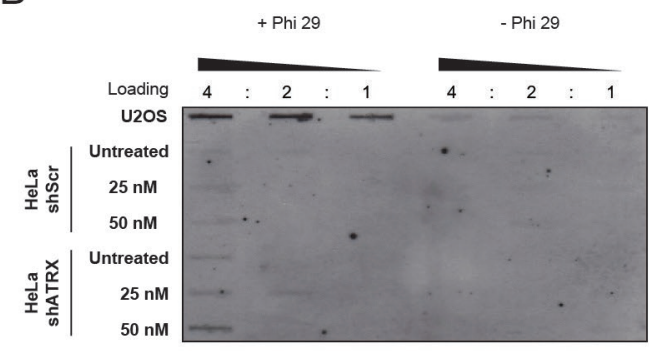

C

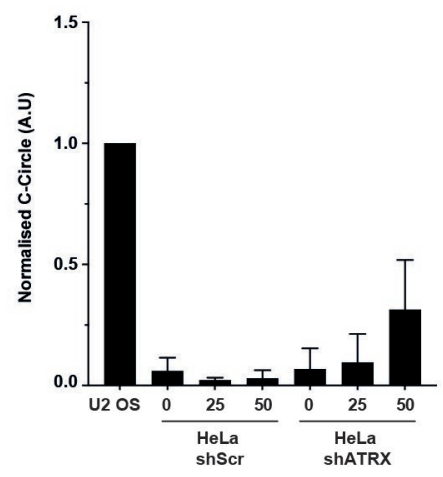

E

shSer NT

shScr + PDS

shATRX NT
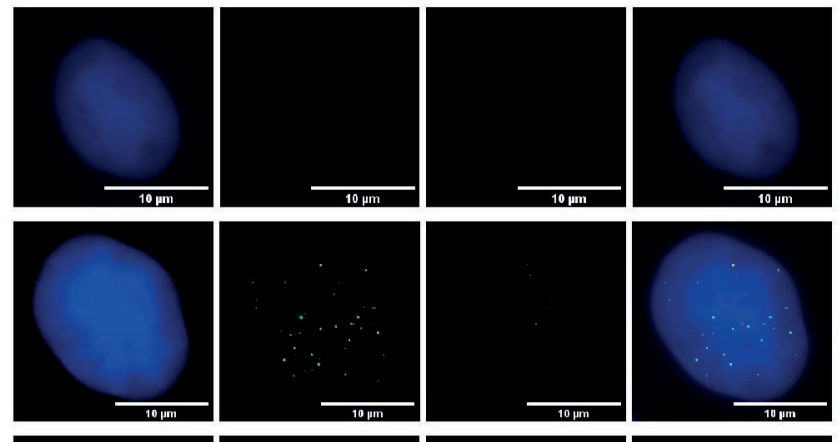
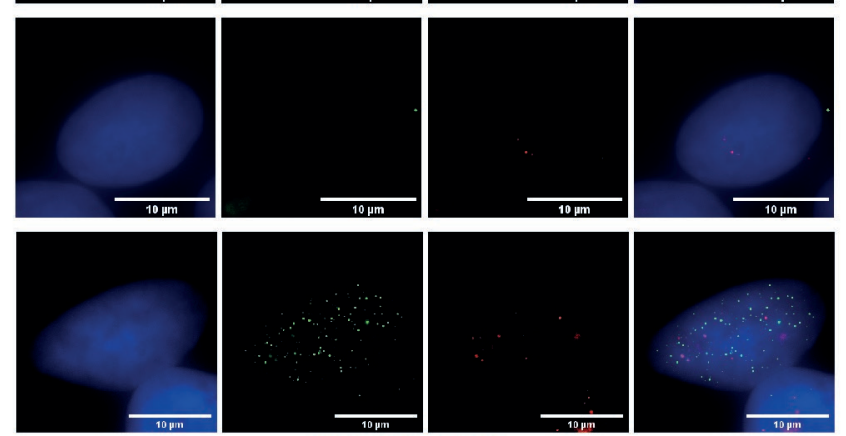

$10 \mathrm{~mm}$

10씨
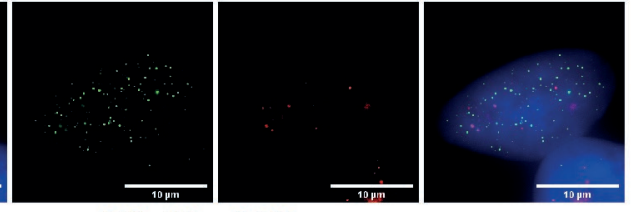
A

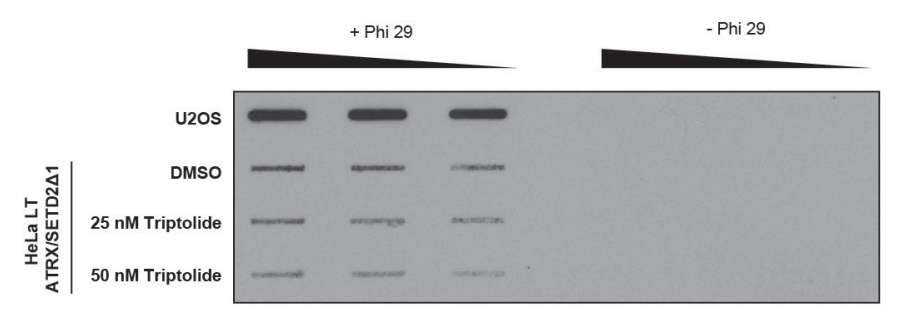

C

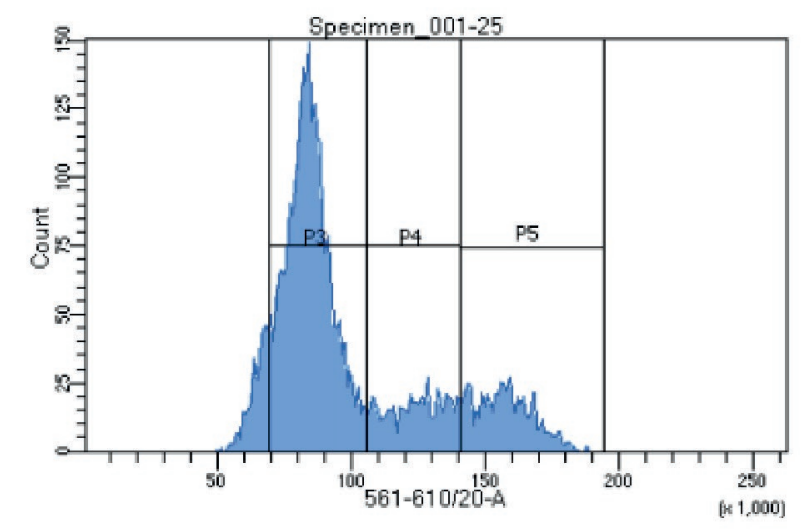

E

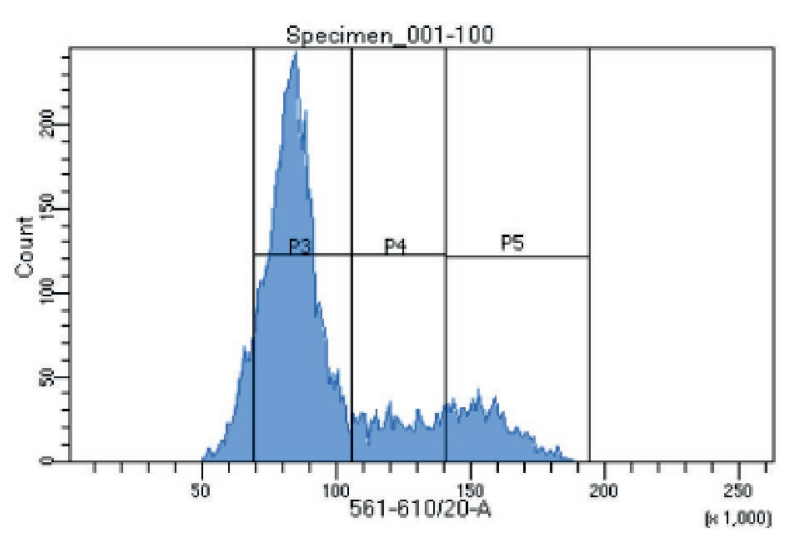

B

HeLa LT ATRX/SETD2 $\triangle 1$ Untreated

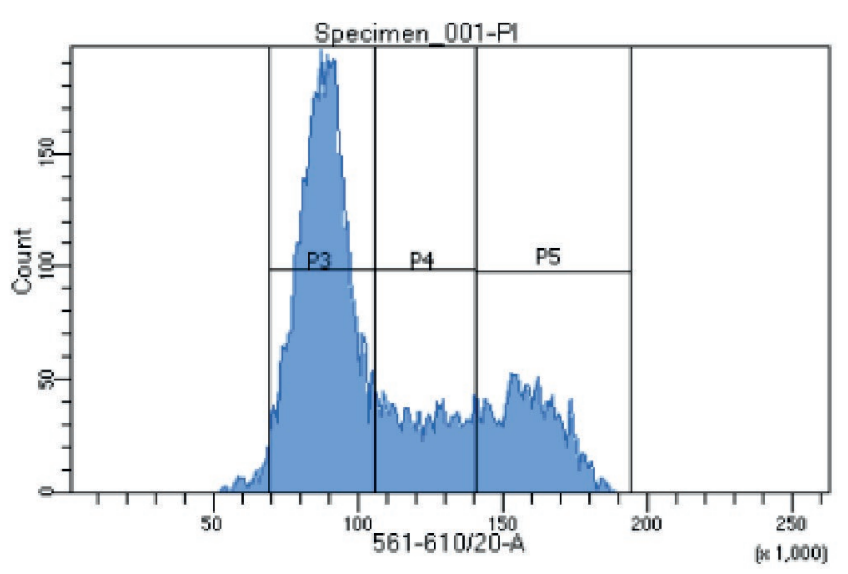

D HeLa LT ATRX/SETD2 $\triangle 150$ nM

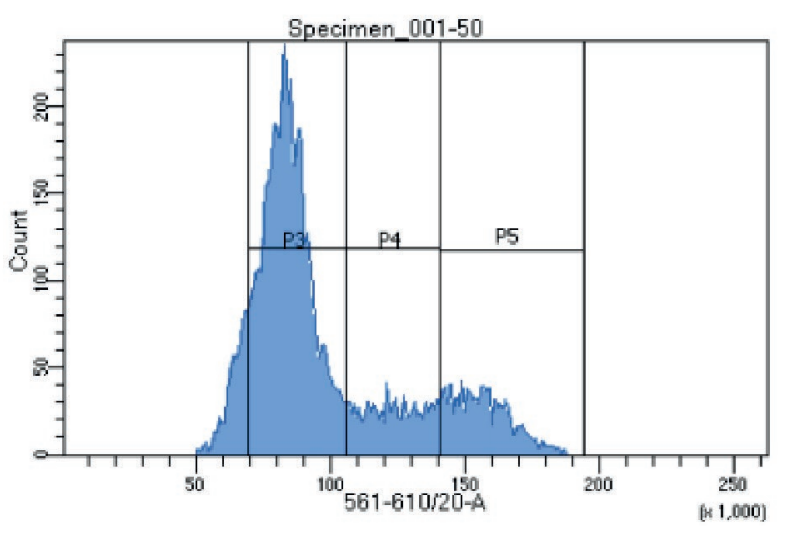

F

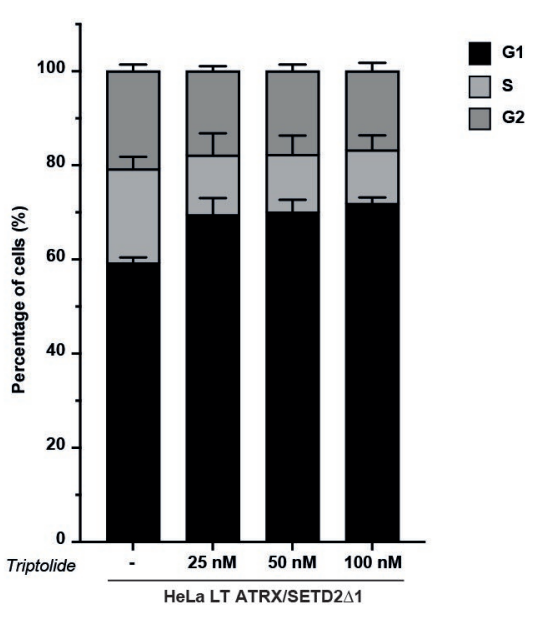


Figures

A

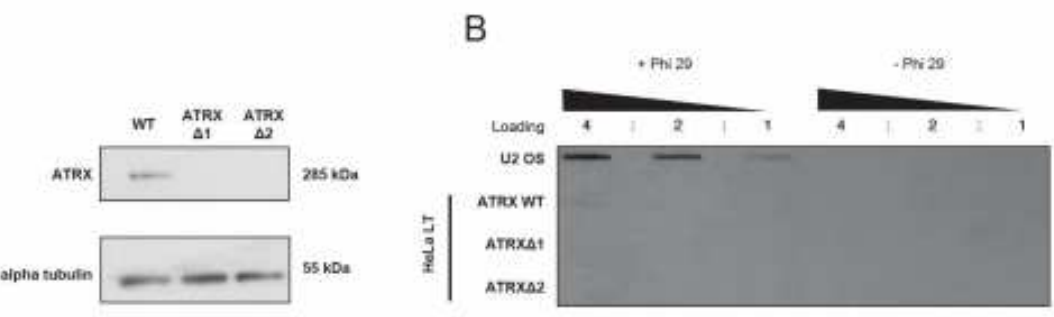

D

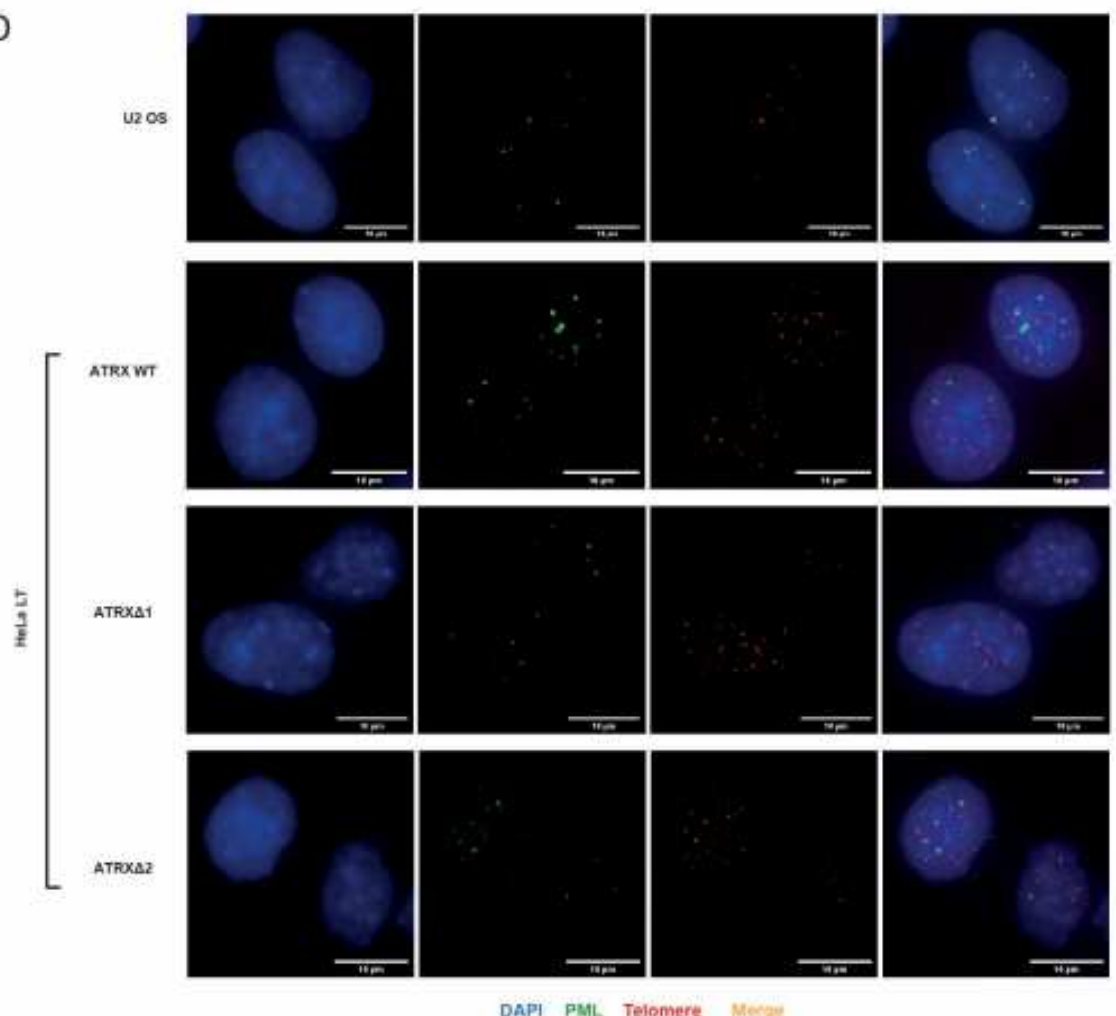

DAPI PML Telomere Meray
C

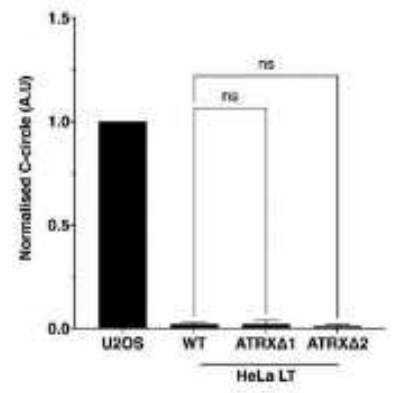

E

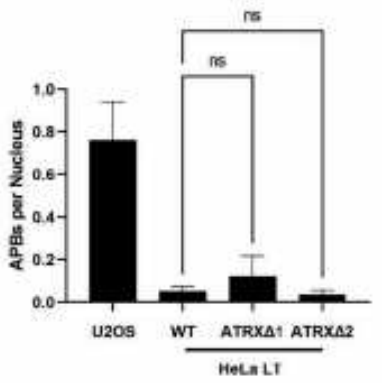

F

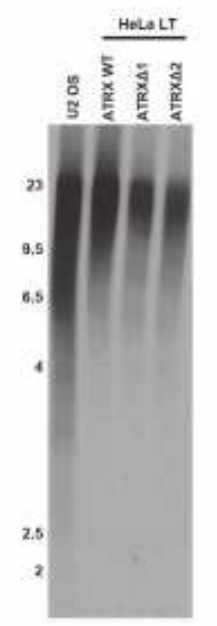

Figure 1

Loss of ATRX alone is insufficient to trigger the ALT pathway. 

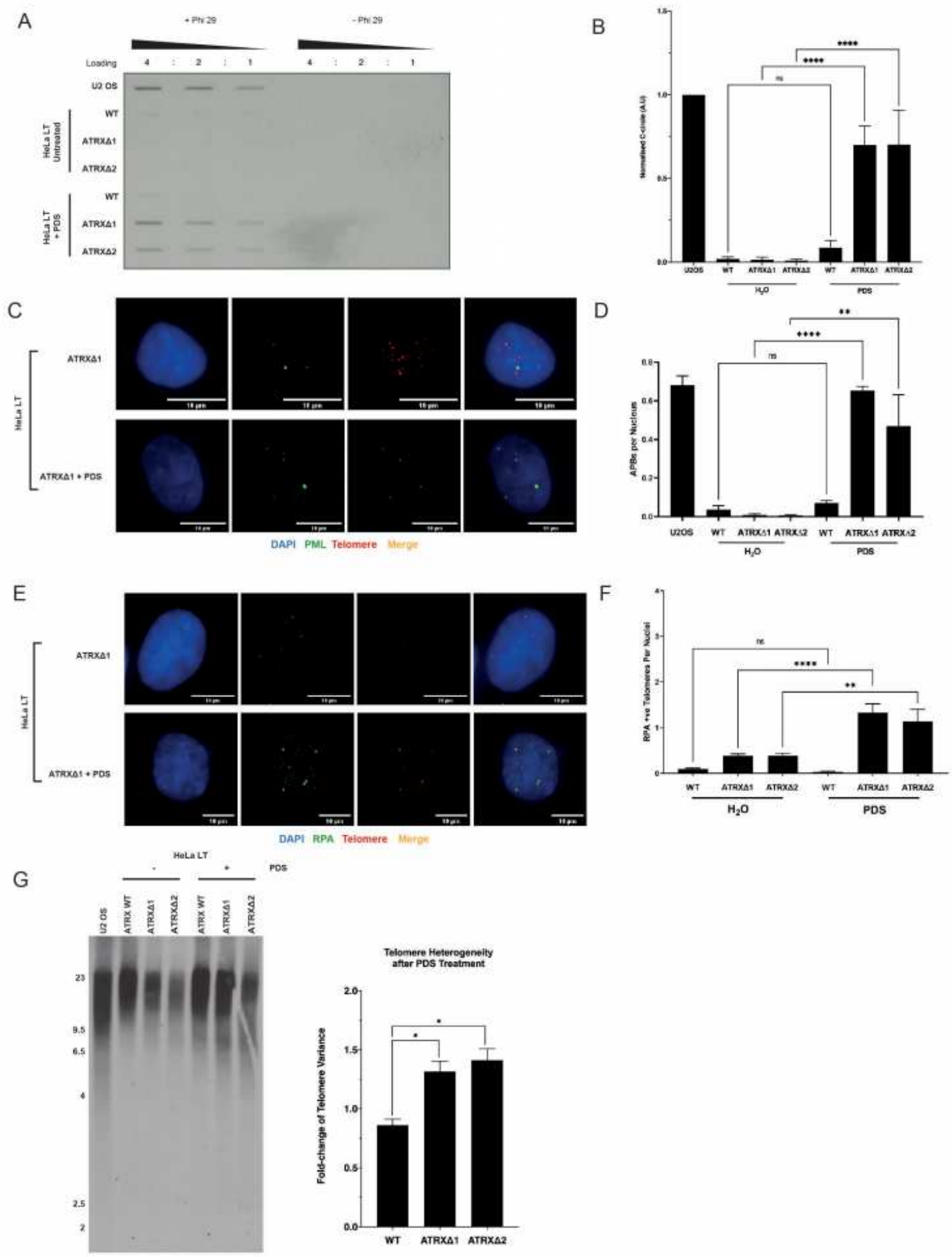

Figure 2

Treatment with PDS in combination with ATRX loss triggers ALT markers. 
A
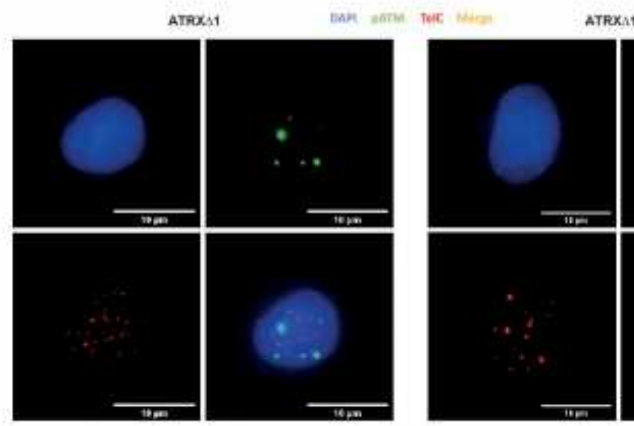

TFXXIt+PDS

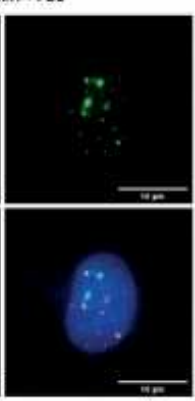

D
B
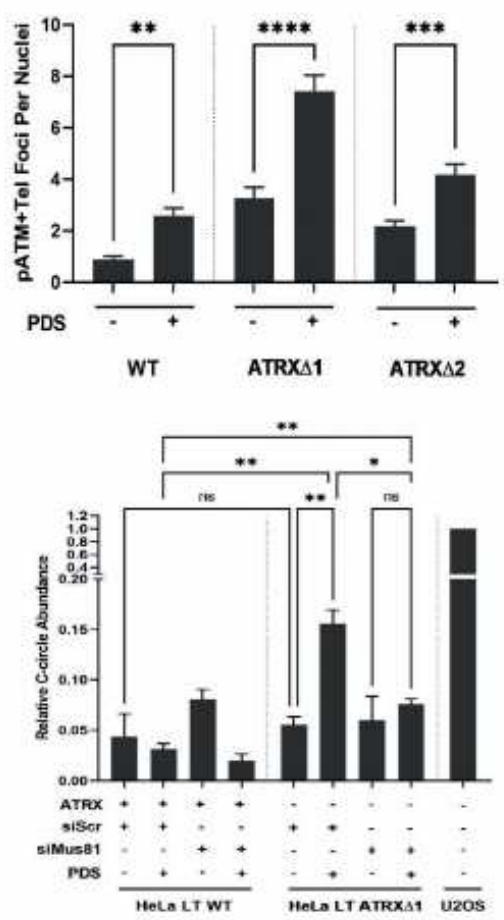

E

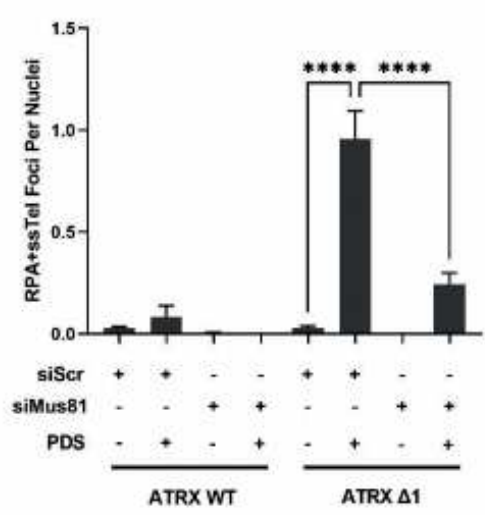

F

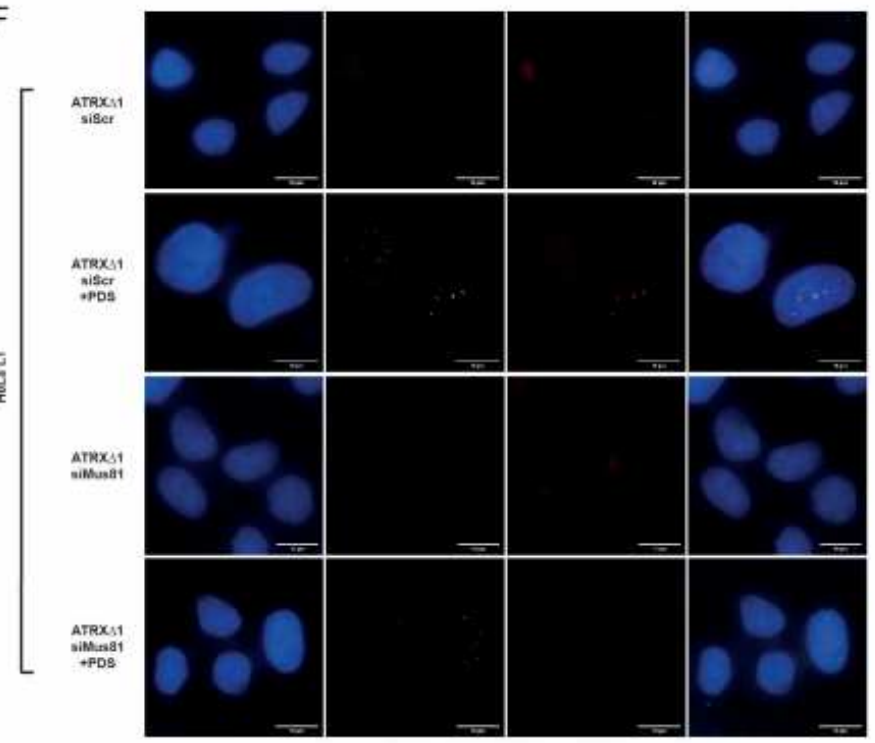

Figure 3

PDS induced ALT markers require replication fork collapse. 
A
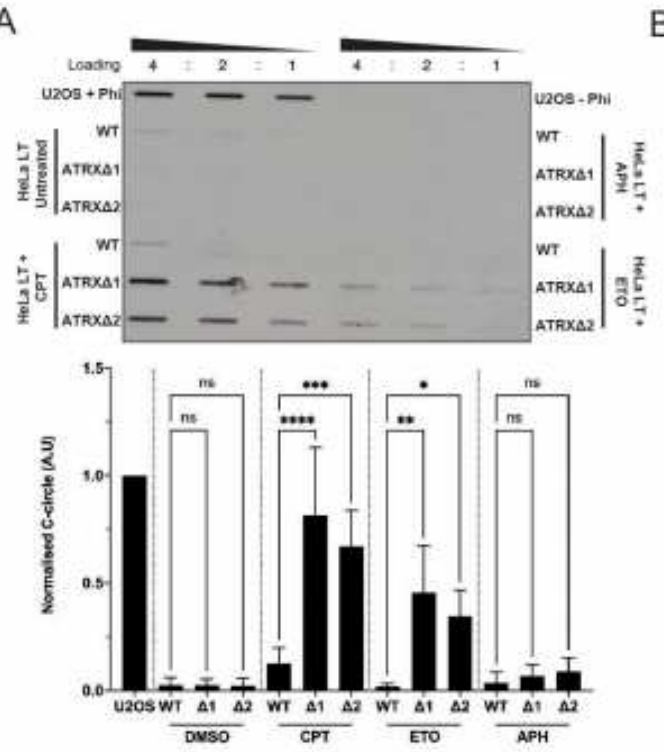

C
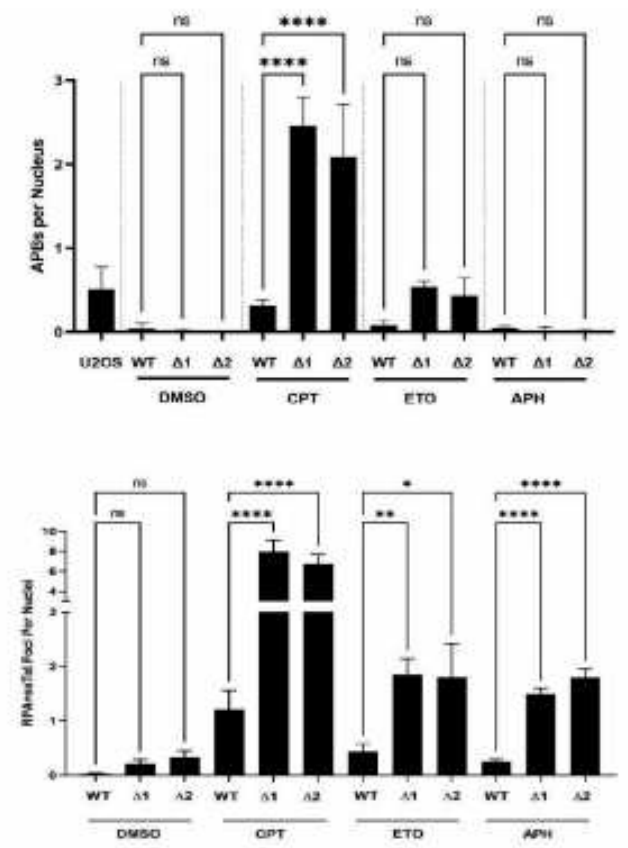

B

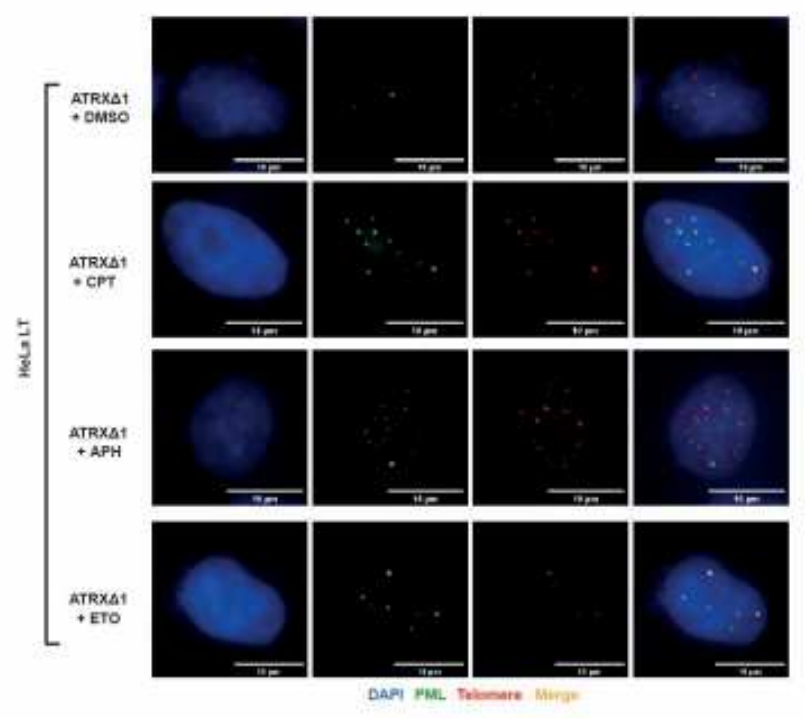

D

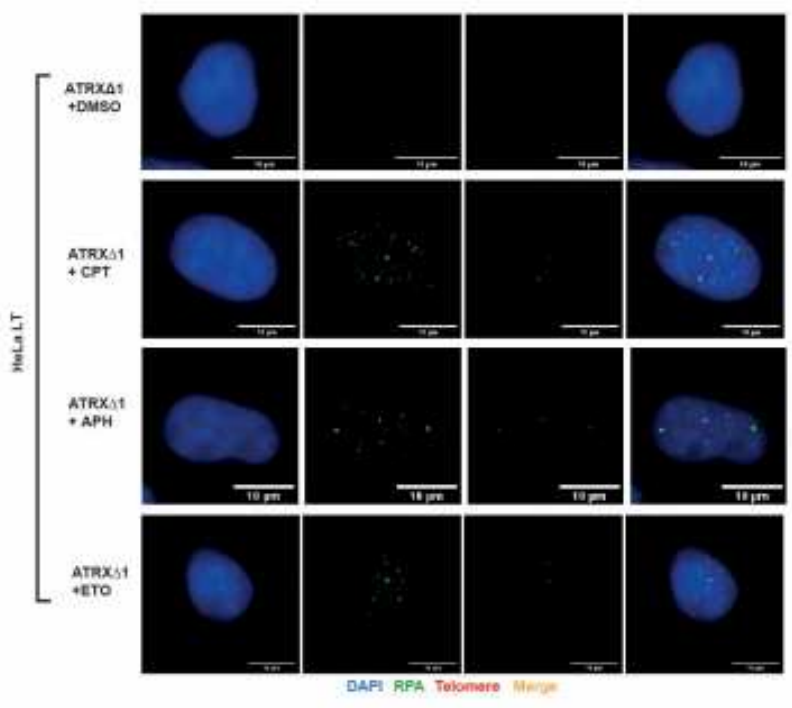

Figure 4

A variety of genotoxic agents induce markers of ALT in ATRX deficient cells. 
A

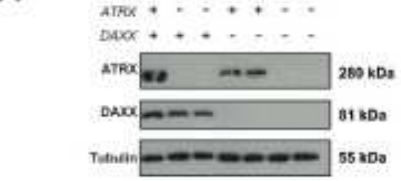

B
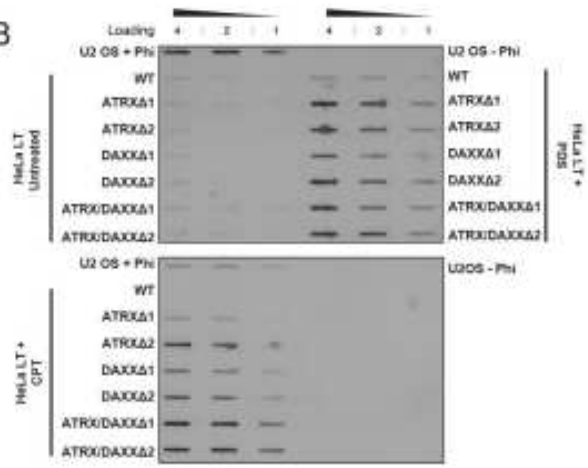

C
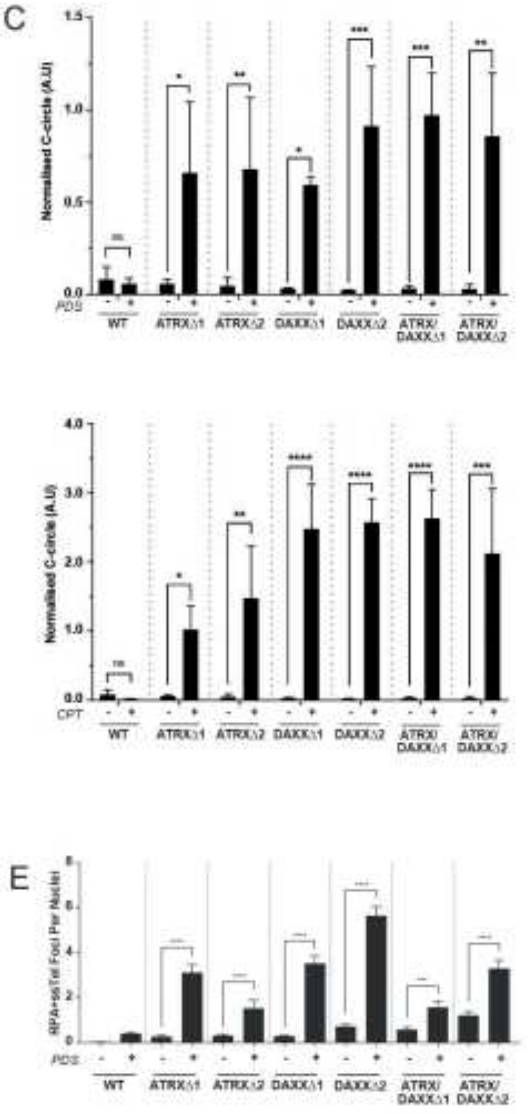

D
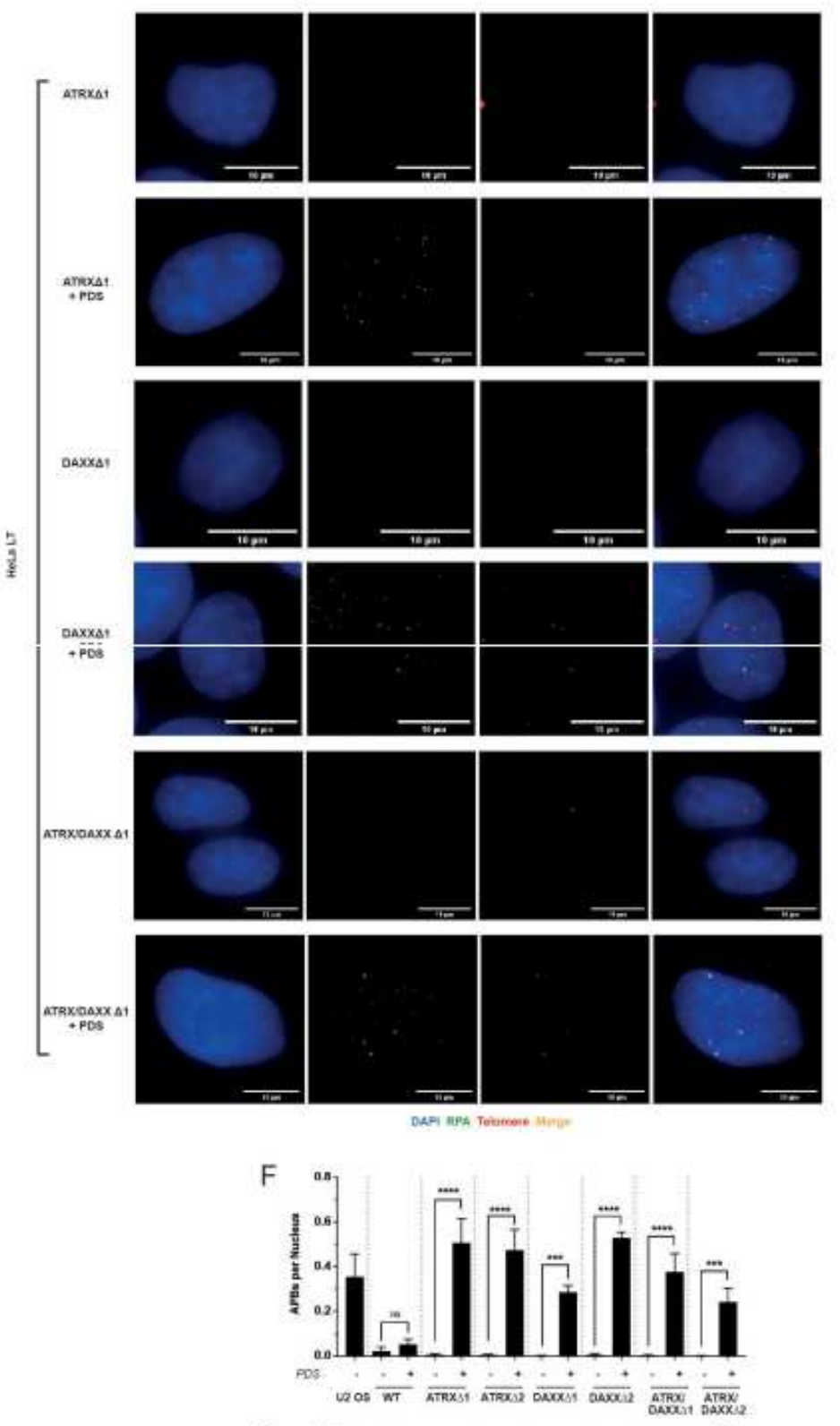

G

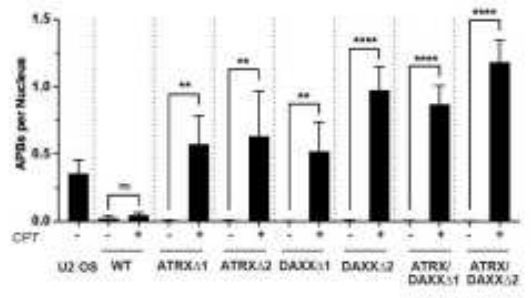

Figure 5

ATRX and DAXX act epistatically in the suppression of induced ALT. 
A

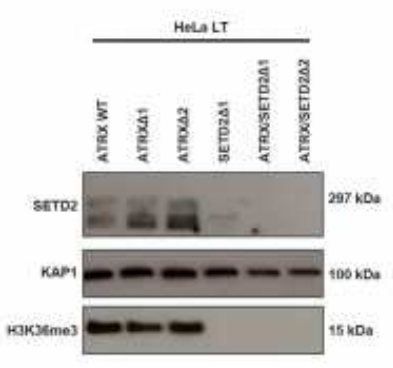

B

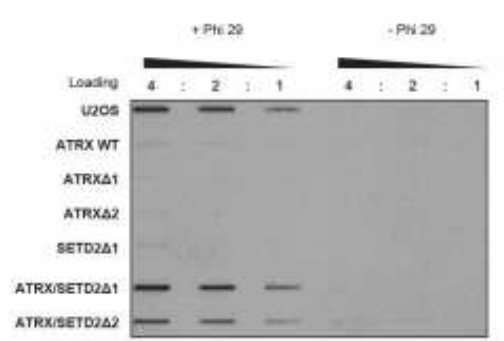

D

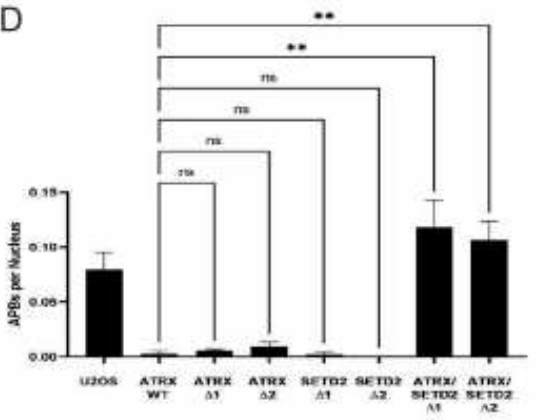

G

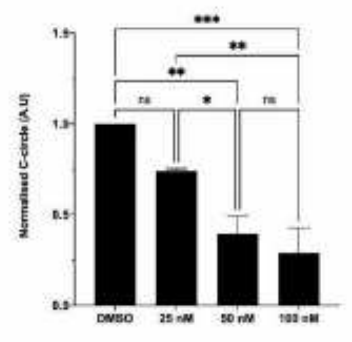

J

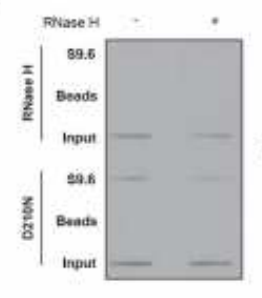

E

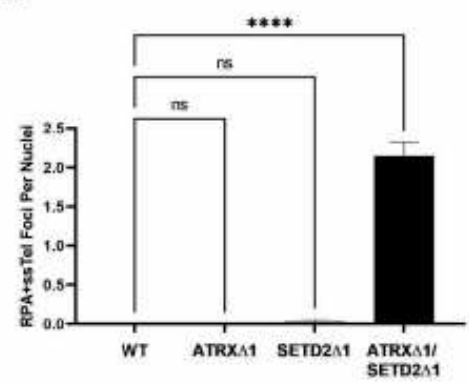

$\mathrm{H}$

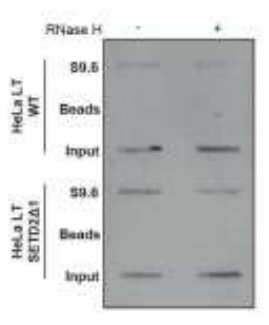

K

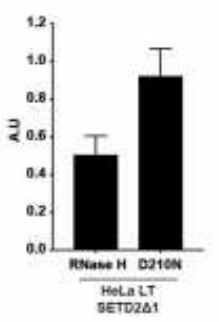

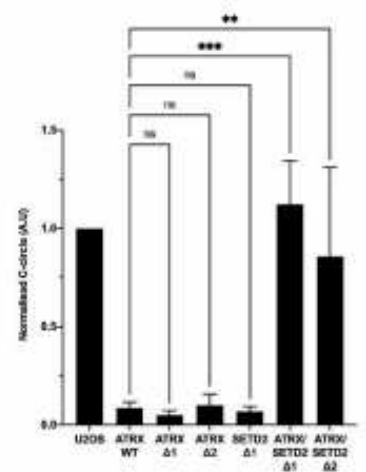

F

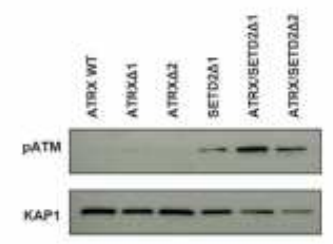

I
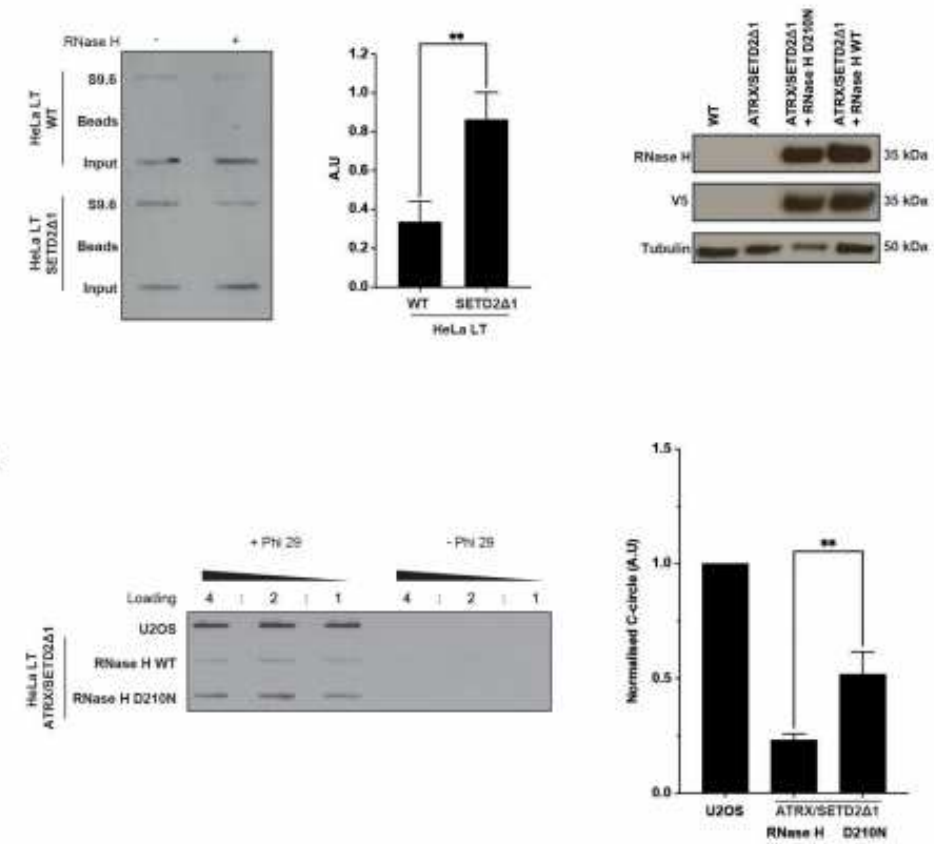

Figure 6

SETD2 loss in combination with ATRX loss triggers markers of the ALT pathway. 
B
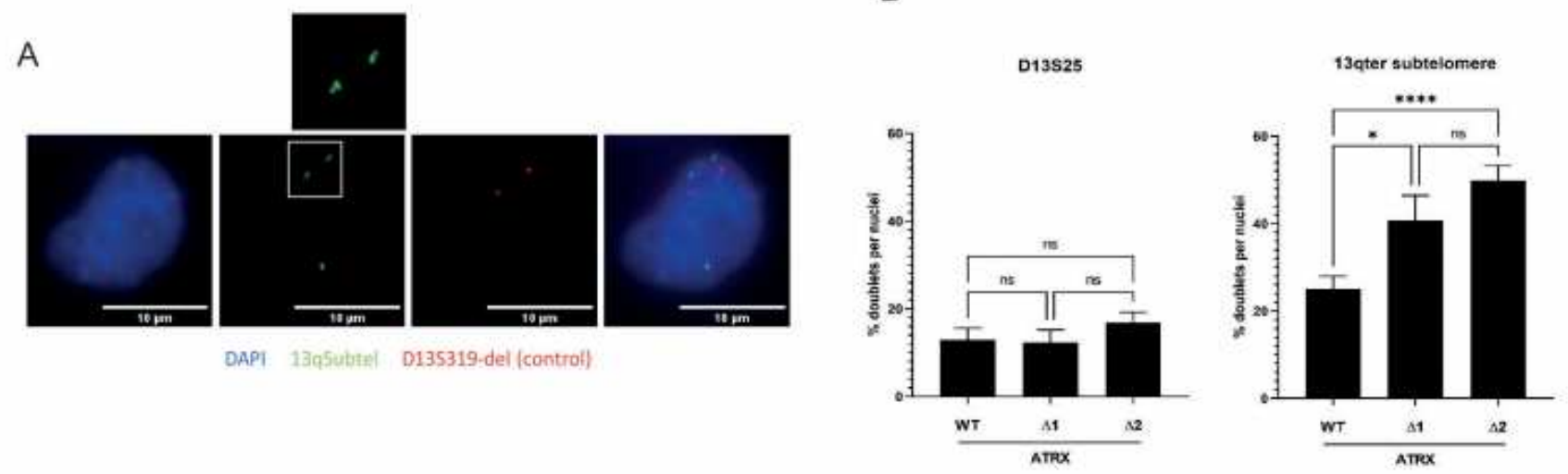

C

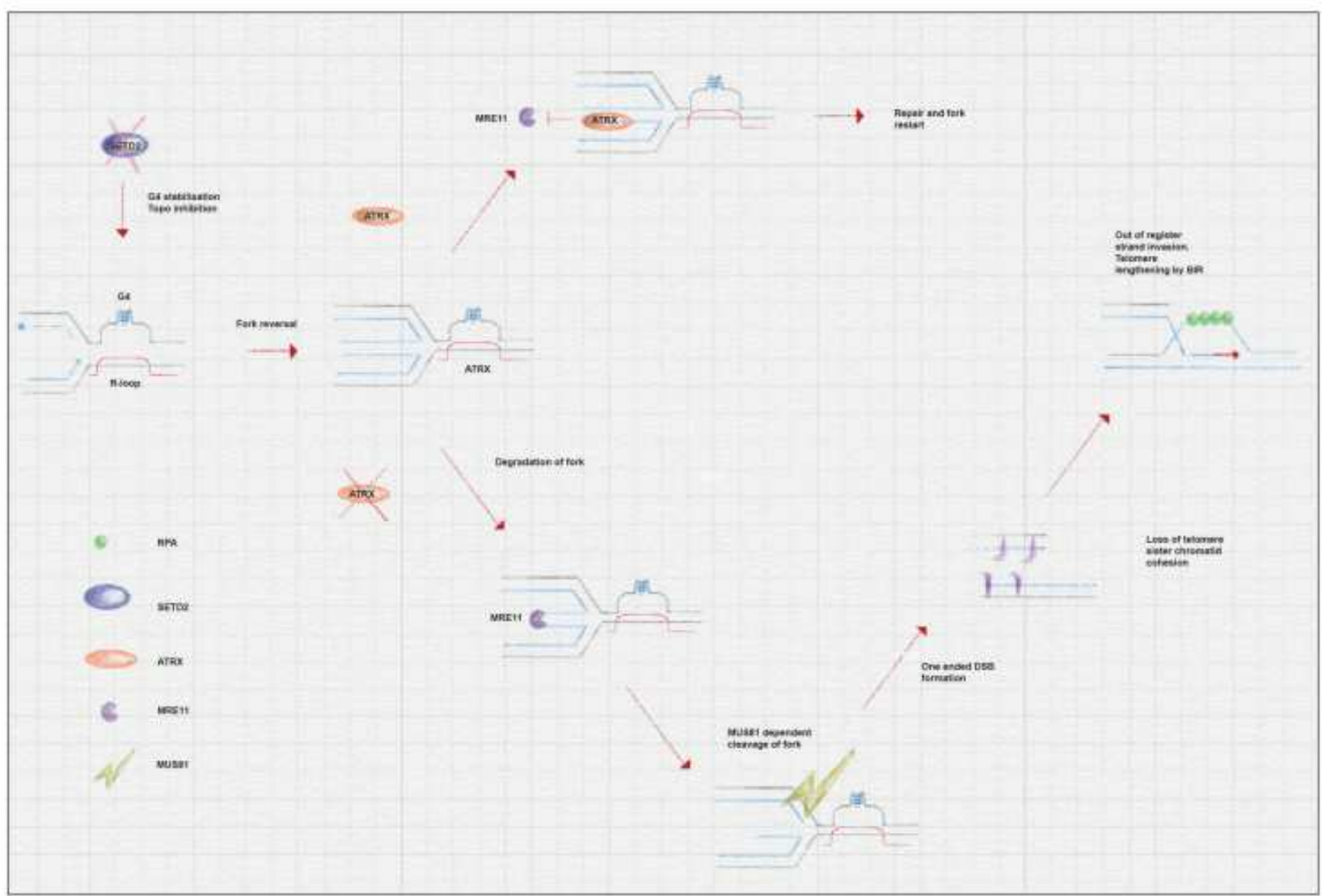

Figure 7

Loss of ATRX induces loss of telomere sister chromatid cohesion. 